\title{
Differential requirements for Gli2 and Gli3 in the regional specification of the mouse hypothalamus
}

\section{OPEN ACCESS}

Edited by:

Agustín González,

Universidad Complutense de Madrid,

Spain

Reviewed by:

Kenji Shimamura,

Kumamoto University, Japan

Pierre-Yves Risold,

Université de Franche-Comté, France

${ }^{*}$ Correspondence:

Gonzalo Alvarez-Bolado,

Department of Medical Cell Biology and Neuroanatomy, University of Heidelberg, Im Neuenheimer Feld 307, Abt. Neuroanatomie (3.OG),

69120 Heidelberg, Germany alvarez@ana.uni-heidelberg.de;

Sandra Blaess,

Laboratory of Neurodevelopmental Genetics, Institute of Reconstructive Neurobiology, Life and Brain Center, University of Bonn, Sigmund Freud Street 25, 53127 Bonn, Germany sandra.blaess@uni-bonn.de;

Luis Puelles,

Department of Morphology, Facultad de Medicina, Instituto Murciano de Investigación Biosanitaria, School of Medicine, University of Murcia, Campus de Espinardo, 30100 Murcia, Spain puelles@um.es

Received: 16 January 2015 Paper pending published: 02 February 2015 Accepted: 09 March 2015 Published: 25 March 2015

Citation: Haddad-Tóvolli R, Paul FA, Zhang Y, Zhou X, Theil T, Puelles L, Blaess S and Alvarez-Bolado G (2015) Differential requirements for Gli2 and Gli3 in the regional specification of the mouse hypothalamus.

Front. Neuroanat. 9:34. doi: 10.3389/fnana.2015.00034

\author{
Roberta Haddad-Tóvolli', Fabian A. Paul' ${ }^{2}$, Yuanfeng Zhang ${ }^{1}$, Xunlei Zhou', \\ Thomas Theil ${ }^{3}$, Luis Puelles ${ }^{4,5 *}$, Sandra Blaess ${ }^{2 *}$ and Gonzalo Alvarez-Bolado ${ }^{1 *}$ \\ ${ }^{1}$ Department of Medical Cell Biology and Neuroanatomy, University of Heidelberg, Heidelberg, Germany, ${ }^{2}$ Laboratory of \\ Neurodevelopmental Genetics, Institute of Reconstructive Neurobiology, Life and Brain Center, University of Bonn, Bonn, \\ Germany, ${ }^{3}$ Centre for Integrative Physiology, University of Edinburgh, Edinburgh, UK, ${ }^{4}$ Department of Morphology, Instituto \\ Murciano de Investigación Biosanitaria, School of Medicine, University of Murcia, Murcia, Spain, ${ }^{5}$ Facultad de Medicina, \\ University of Murcia, Murcia, Spain
}

Secreted protein Sonic hedgehog (Shh) ventralizes the neural tube by modulating the crucial balance between activating and repressing functions (GliA, GliR) of transcription factors Gli2 and Gli3. This balance-the Shh-Gli code-is species- and contextdependent and has been elucidated for the mouse spinal cord. The hypothalamus, a forebrain region regulating vital functions like homeostasis and hormone secretion, shows dynamic and intricate Shh expression as well as complex regional differentiation. Here we asked if particular combinations of Gli2 and Gli3 and of GliA and GliR functions contribute to the variety of hypothalamic regions, i.e., we wanted to approach the question of a possible hypothalamic version of the Shh-Gli code. Based on mouse mutant analysis, we show that: (1) hypothalamic regional heterogeneity is based in part on differentially stringent requirements for Gli2 or Gli3; (2) another source of diversity are differential requirements for Shh of neural vs. non-neural origin; (3) the medial progenitor domain known to depend on Gli2 for its development generates several essential hypothalamic nuclei plus the pituitary and median eminence; (4) the suppression of Gli3R by neural and non-neural Shh is essential for hypothalamic specification. Finally, we have mapped our results on a recent model which considers the hypothalamus as a transverse region with alar and basal portions. Our data confirm the model and are explained by it.

Keywords: embryo, Gli1, Gli2, Gli3, hypothalamus, mouse, mutant, Shh

\section{Introduction}

Sonic hedgehog (Shh) is a morphogen required for ventral neural tube specification (Echelard et al., 1993; Ericson et al., 1995, 1997; Chiang et al., 1996). Shh acts through the Gli transcriptional activators (GliAs) and repressors (GliRs); the balance between GliA and GliR specifies ventral differentiation and proliferation (Lee et al., 1997; Ruiz i Altaba, 1997; Brewster et al., 1998). This "Shh-Gli code" is known for the mouse spinal cord [reviewed in Ruiz i Altaba et al. (2003), Dessaud et al. (2008), Ingham et al. (2011)] and brainstem (Wang et al., 1995; Blaess et al., 2006, 2011; Feijoo et al., 2011).

The Shh expression domain in the forebrain is more extensive and elaborate than in the spinal cord, has become more intricate and dynamic during phylogenesis and is considered a motor of 
brain evolution (Osorio et al., 2005). Work on thalamic development supports the notion that regional variation of the ShhGli code underlies forebrain complexity (Haddad-Tovolli et al., 2012). In the same way, the canonical Shh-Gli code shows interspecies variation (Ruiz i Altaba, 1997; Aberger and Ruiz, 2014). On the basis of mutant phenotype analysis at different rostrocaudal levels of the mouse spinal cord and the hindbrain it has been proposed that Gli2 and Gli3 have partially overlapping roles and that their relative contributions to ventral specification shows regional variation (Motoyama et al., 2003; Lebel et al., 2007).

The hypothalamus regulates homeostasis, endocrine secretion, and reproductive behavior (Saper, 2006; Puelles et al., 2012; Sternson, 2013) and its alterations can cause conditions like obesity and high blood pressure (Caqueret et al., 2005; McMillen et al., 2008). Complex gene expression pattern combinations underlie hypothalamic regional specification (Shimogori et al., 2010; Puelles et al., 2012). On the basis of classical neuroanatomy studies, the adult hypothalamus has been traditionally described as subdivided into four regions (preoptic, anterior, tuberal, and mamillary) arranged rostro-caudally and ventrally in the brain (Figure 1A) and flanked by the lateral hypothalamic area (LHA), a large region essential to regulate behavioral state and arousal (Swanson, 1987). The modern view considers the adult hypothalamus as part of a behavioral control column (Swanson, 2000).

A

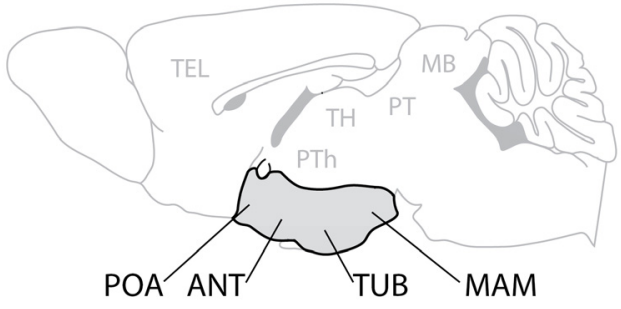

B

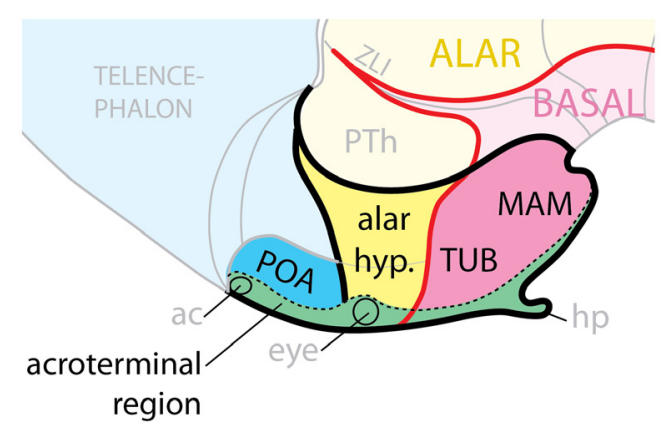

FIGURE 1 | Hypothalamic regions. (A) Conventional representation of the hypothalamus (gray) as ventral region with four rostro-caudal regions, POA, preoptic; ANT, anterior; TUB, tuberal; MAM, mamillary. (B) Model of the hypothalamus considering Shh expression (pink) as basal (ventral) marker. The POA is part of the telencephalon; the alar hypothalamus (yellow) corresponds to the anterior region; the tuberal and mamillary regions are not "caudal" but basal (ventral). ac, anterior commissure; hp, hypophysis; PTh, prethalamus; ZLI, zona limitans.
Here we analyze the hypothalamic phenotypes of mouse mutants to ascertain which combinations of GliA and GliR specify the mouse hypothalamic regions and which Gli genes perform these functions. We examined embryos primarily after midgestation so that we could assess which hypothalamic nuclei are affected when the GliA/R code is affected. We have mapped our results on a model of the developing hypothalamus (Puelles et al., 2012; Figure 1B) built around the observation that, since Shh is indispensable to ventralize the neural tube, and it is expressed in a long domain stretching the entire length of it, it follows that during development the Shh expression boundary separates dorsal (alar) from ventral (basal). The rostral end of the developing neural tube is closed by a transverse structure called acroterminal region, which does not share the typical characteristics of the floor plate. Beyond mamillary level, the acroterminal region extends all the way through the tuberal region, alar hypothalamus and preoptic region and up to the anterior commissure, it is transversally oriented (has alar and basal portions) and strongly patterned (probably by the underlying prechordal plate) and would generate the median eminence, infundibulum, neurophypophysis, preoptic terminal lamina, eyes, optic chiasma, and suprachiasmatic area. Two progenitor domains, medial and lateral, give rise to the basal part of the hypothalamus: the medial domain generates median eminence and neurohypophysis, medial portions of the ventromedial and arcuate nuclei, and the mamillary body; the lateral originates most of the ventromedial nucleus, the dorsomedial nucleus and the LHA (Alvarez-Bolado et al., 2012).

We show that, in the basal hypothalamus, the medial progenitor domain requires non-neural Shh acting through Gli2A. The lateral progenitor domain is patterned by neural Shh acting through Gli3R and Gli2A or Gli3A. In the presence of Shh signaling, the Gli3R function is not required for hypothalamus specification. Neither Gli2 nor Gli3 are required for overall patterning of the alar hypothalamus and preoptic area. These data confirm the main tenets of the model (Puelles et al., 2012), since they strongly support a subdivision of the developing hypothalamus into alar and basal domains.

\section{Materials and Methods}

\section{Mice and Mouse Lines}

Animals were housed and handled in ways that minimize pain and discomfort, in accordance with German animal welfare regulations (TierSchG) and in agreement with the European Communities Council Directive (2010/63/EU). The authorization for the experiments was granted by the Regierungspräsidium Karlsruhe (state authorities) and the experiments were performed under surveillance of the Animal Welfare Officer responsible for the Institute of Anatomy and Cell Biology. To obtain embryos, timed-pregnant females were sacrificed by cervical dislocation; the embryos were decapitated.

\section{Gli2 $^{z f d /+}$ (Gli2 Zinc Finger-Deleted) Mutant Mice}

This Gli2 null mutant mouse line was generated (Mo et al., 1997) by replacing the exons encoding for zinc fingers $3-5$. The deletion 
leads to an out-of-frame mutation causing disrupted transcription from the deletion site to the 3 end of the Gli2 gene. This results in translation of a truncated protein unable to bind to DNA, since the zinc fingers 4 and 5 are essential for DNA binding (Pavletich and Pabo, 1993). The Gli2 $2^{z f d / z d f}$ are null mutants for Gli2; the Gli2 $2^{z f d /+}$ have normal phenotypes and are used as controls.

\section{Gli3 $^{X t-J /+}$ (Extra-Toes) Mutant Mice}

This line carries a $50 \mathrm{~kb}$ deletion that removes the exons encoding zinc fingers 3-5 and the complete $3^{\prime}$ part of the Gli3 gene (Hui and Joyner, 1993; Maynard et al., 2002; Genestine et al., 2007). The Gli3 ${ }^{X t-J / X t-J}$ are null mutants for Gli3.

We have not been able to obtain double Gli2-Gli3 mutant embryos ( $n=4$ litters, 1 at E9.5, 2 at E10.5, and 1 at E12.5).

\section{Gli3-nlacZ Mutant Mice}

The Gli3-nlacZ knock-in mouse line was generated by partially replacing the first coding exon of Gli3 with the nlacZ cDNA. Thus, expression of lacZ is controlled by the endogenous Gli3 promoter/enhancer elements and can be used to monitor the expression pattern of Gli3 (Garcia et al., 2010).

\section{Foxb1-Cre Mutant Mice}

Express Cre in the thalamic and hypothalamic neuroepithelium (Zhao et al., 2007, 2008). We used only heterozygous Foxb1-Cre mice, which show a normal phenotype (Zhao et al., 2007, 2008), Foxb1 Cre/Cre homozygotes were not used in this study.

\section{Foxb1-Cre;Shh ${ }^{f /+}$ Mutant Mice}

To obtain mice specifically deficient in Shh expressed in the neural tube (conditional knock-out for neural Shh), we crossed our Foxb1-Cre mice (Zhao et al., 2007, 2008) with Shhf/+ conditional mutants in which exon 2 of the Shh gene was flanked by loxP sites (Dassule et al., 2000; Lewis et al., 2001). The Shhf/+ conditional mutants were generated in the laboratory of Dr. Andrew McMahon (University of Harvard) and were obtained through Jackson Labs (www.jax.org). The Foxb1-Cre;Shh ${ }^{f / f}$ mutants lack all Shh expression in the forebrain neuroepithelium (Szabo et al., 2009a,b).

\section{Foxb1-Cre;Shh ${ }^{f /+} ; \mathrm{Gli}^{X t-J /+}$ Mutant Mice}

The double homozygous mutants for Shh expressed in the neural tube (neural Shh or nShh) and Gli3 were generated by crossings between Foxb1Cre;Shh $f /+$ mice; and Gli3 ${ }^{X t-J /+}$ mice. The double mutants (Foxb1-Cre;Shh ${ }^{f / f} ; \mathrm{Gli3}^{\mathrm{Xt-J/Xt-J}}$ ) do not survive beyond birth.

\section{In Situ Hybridization}

Embryos or embryonic brains were dissected, fixed in $4 \%$ paraformaldehyde, and embedded in paraffin. Non-radioactive ISH was performed on paraffin sections (7 $\mu \mathrm{m}$ for E10.5, $10 \mu \mathrm{m}$ for E12.5, and $14 \mu \mathrm{m}$ for E18.5 embryos) that were fixed in $4 \%$ paraformaldehyde and acetylated after sectioning. RNA in situ hybridization was performed as described (Blaess et al., 2011).

\section{BrdU Labeling}

Pregnant mice (E12.5) from appropriate crossings were injected intraperitoneally with $5^{\prime}$-bromo- $2^{\prime}$-deoxyuridine (BrdU; Sigma; $50 \mu \mathrm{g} / \mathrm{g}$ body weight) at 12:00 h. Three hours after the injection, embryos were collected and fixed overnight in $4 \%$ PFA in PBS at $4^{\circ} \mathrm{C}$. Cell proliferation was detected by means of antibody (rat anti-BrdU; AbCam; 1:100) after epitope retrival in Tris-EDTA buffer $\mathrm{pH}=9.0$ for $20 \mathrm{~min}$ in pressure cooker. The nuclear marker 4'6-diamidino-2-phenylindole dihydrochloride (DAPI; Invitrogen) was used as a counterstain. For cell counting, $10 \mu \mathrm{m}$ paraffin sections were analyzed under a confocal microscope (LSM700 -Zeiss) and DAPI and BrdU-positive cells were counted in 100- $\mu \mathrm{m}$-wide bins encompassing the thickness of the neuroepithelium (apical to basal side) at four hypothalamic sites (preoptic area, alar hypothalamus and tuberal and mamillary regions) on two histological sections per level in three animals per age and genotype (WT, Gli2 $2^{z f d} / z f d, G l i 3^{X t-J / X t-J}$, and Foxb1-Cre;Shh ${ }^{f /+}$;Gli3 ${ }^{\mathrm{Xt}-\mathrm{J} /+}$ double mutants). The BrdUlabeling index (BrdU-labeled cells as percentage of total cells) was then calculated (Takahashi et al., 1993; Warren et al., 1999; Ishibashi and McMahon, 2002).

\section{Cloning of Constructs}

In an expression vector driven by pCAGGS (Niwa et al., 1991) we inserted either EmGFP (kind gift of Dr. Boris Fehse, University of Hamburg; Weber et al., 2010) or tdTomato (kind gift of Dr. Roger Y. Tsien, UCSD) as reporters. On vectors carrying the tdTomato reporter we then inserted (upstream an internal ribosomal entry site and the reporter) a mutated form of human PTCH1 in which we deleted part (between MfeI and NsiI) of the second large extracellular loop (PTCH1- $\Delta$-loop2), as was done in Briscoe et al. (2001).

\section{In Utero Electroporation}

This procedure was carried out as described (Saito and Nakatsuji, 2001; Saito, 2006; Haddad-Tovolli et al., 2012) with added caveats for hypothalamus targeting (Haddad-Tovolli et al., 2013). Pregnant mice at E12.5 were anesthetized with a mixture of Halothane (Isoflurane, Baxter) and oxygen $(0.5 \mathrm{l} / \mathrm{min})$ administered with a Komesaroff Anaesthetic Machine. The uterus was exposed and the DNA solution $(1 \mu \mathrm{g} / \mu \mathrm{l})$ was injected with a glass micropipette in the third ventricle of the embryo brain through the uterine wall. Electric pulses were administered with a CUY21 electroporator (Nepagene, Japan; 5 square-wave pulses, $50 \mathrm{~V}, 50 \mathrm{~ms}$ on/950 ms off) and a stainless steel needle electrode (CUY550-10) used as positive pole and a round flat electrode (CUY700P4L) as negative pole. After the surgery, the embryos were allowed to develop in utero for 6 days and collected at E18.5 for analysis. The embryonic brains were dissected, fixed overnight in $4 \% \mathrm{PFA}$ in $\mathrm{PBS}$ at $4^{\circ} \mathrm{C}$ and then protected with sucrose (20; $30 \%$ ) and embedded in OCT mounting medium (Tissue Tek). Blocks were sectioned into $20 \mu \mathrm{m}$ thick sections in a cryostat (Leica CM3050S) and observed and photographed with a Zeiss LSM 700 confocal microscope. We used laser line $488 \mathrm{~nm}$ for the green reporter EmGFP (excitation maximum $487 \mathrm{~nm}$, emission maximum $509 \mathrm{~nm}$ ) and laser line $555 \mathrm{~nm}$ for the red reporter tdTomato (excitation maximum 554, emission maximum 581). 
Since our readouts are based in the comparison between numbers of cells counted on confocal images (see next paragraph), it was imperative to relie on strictly comparable data. To guarantee comparability, the images of experimental and control brains were obtained under the exact same conditions and with the exact same confocal settings.

\section{Experimental Design of In Utero Electoporation Experiments}

Because each in utero electroporation experiment results in a different number of neuroepithelial cells being transfected, the experiments are not directly comparable with each other. For this reason we do "two-reporter-experiments" (Haddad-Tovolli et al., 2012). The two reporters answer two problems. The green reporter construct (GFP) is an internal control. It will label every one of the transfected neuroepithelial cells and their progeny. In this way, for each single electroporated mouse embryo we know how many cells have been transfected. The second question is the actual scientific question: "does Ptch1-delta-loop2 reduce proliferation?" For this, we have a second construct expressing a dominant loss-of-function version of the Ptch1 receptor (Ptch1delta-loop2, see above Cloning of constructs) and, in the same construct, a red reporter (tandem dimer tomato, tdTomato). We use a ratio of 2 (GFP):1 (Ptch1-delta-loop2+red) in order to introduce some bias in the results, so that the readout of the experiment is the ratio between green cells and red cells. In principle there must be, after electroporation, a very few cells which are only green: they happen to express only GFP (not Ptch1-delta-loop2+red), proliferate normally and generate numerous green neurons, otherwise presumably normal. If the cells coexpressing the green plus the red (= experimental) constructs proliferate less, we will see less green + red neurons.

In parallel, we performed control experiments transfecting a 2:1 mixture of GFP construct and tdTomato construct (without loss-of-function Ptch1 protein) in order to evaluate how many only green and how many green-plus-red neurons we obtain in normal circumstances (i.e., without introducing any dominant loss-of-function). Those are the gray bars in Figure 10J. Additionally, these control experiments remove a possible concern related to the relative brightness of the green and the red reporters. In principle, a green cell could have been transfected also with some red (experimental) constructs in a number to small to be detected (since EmGFP is brighter than tdTomato). This possible source of imprecision can be disregarded since our readout is not absolute but relative (comparison between gray bars and black bars; Figure 10J).

\section{Statistics}

Statistical assessment of the BrdU and electroporation data was performed with Prism 6 software (Graph Pad Software, San Diego, CA, USA).

\section{Morphological Interpretive Model}

The results of mutant analysis were interpreted and mapped using the updated prosomeric model (Puelles et al., 2012) and the Allen Brain Atlas (Allen-Institute-for-Brain-Science, 2009).

\section{Results}

\section{Developing Hypothalamic Expression of Shh and Gli can be Broadly Subdivided into at least Three Stages}

Our purpose was to determine for each of the mouse hypothalamic regions which member of the Gli family performs the GliA and which one the GliR function, and which combinations of GliA and GliR specify these regions-in short, the hypothalamic Shh-Gli code. The expression of Gli1, Gli3, and Shh has been assessed at several stages in the developing chick hypothalamus, but in mouse the data are less comprehensive (Aoto et al., 2002; Ohyama et al., 2008). Thus, the first requisite for our study was to ascertain a detailed spatial-temporal expression map for the three mammalian Gli genes and Shh in the developing hypothalamus of the mouse (Figures 2 and 3). Although inactivation of Gli1 does not result in an abnormal phenotype (Park et al., 2000; Bai et al., 2002), Gli1 expression is a readout for Shh signaling [see references in Lewis et al. (2001)] and for this reason it was important to analyze its expression domain too. It has been described that, in the mouse neural plate, expression of Gli genes is first detected at E7.5 (neural fold); in this early stage of Gli expression, Gli1 is expressed only in the midline of the neural fold, while Gli2 and Gli3 expression is widespread in the entire ectoderm (Hui et al., 1994) and Shh is expressed in the underlying mesoderm (non-neural Shh; Echelard et al., 1993).

We started our investigation of Gli expression patterns after neurulation, when they become more complex and at the same time more relevant to our study. At E8.5 (middle stage; Figure 2), Gli1 and Gli2 were expressed in overlapping patterns in the lateral domain (Alvarez-Bolado et al., 2012; Figures 2A,B), while Gli3 was expressed in a more peripheral, non-hypothalamic domain (Figure 2C) and Shh was expressed in the medial domain (neural Shh, medial expression; Figure 2D), in coincidence with the medial progenitor domain (Alvarez-Bolado et al., 2012). The presumptive hypothalamus was defined by expression of specific marker Nkx2-1 (Figures 2E,J).

At E10.5 (late stage; Figure 3) the Gli expression pattern had changed again. While Gli2 expression was absent from the hypothalamic primordium (Figures 3G-I), Gli3 and Shhactivation diagnostic marker Gli1 showed overlapping expression domains in the medial domain (Figures 3A-C,M-O), suggesting a potential activator function of Gli3 (Gli3A) in the midline at this age. Shh was expressed in a lateral domain corresponding to the lateral progenitor domain (Alvarez-Bolado et al., 2012; neural Shh, lateral expression). We concluded that the hypothalamic expression of Shh and the Gli genes can be broadly subdivided into at least three stages (summarized in Figure 11A).

\section{Deficiency in Gli2 or Gli3 does not Alter the Overall Specification of the Alar Hypothalamus}

Sonic hedgehog is required to specify hypothalamic structures and the preoptic area (Chiang et al., 1996; Pabst et al., 2000; Rallu et al., 2002). In mouse mutants lacking Shh expression in the neural tube (Foxb1-Cre;Shh ${ }^{f / f}$ mutants), however, the preoptic and 

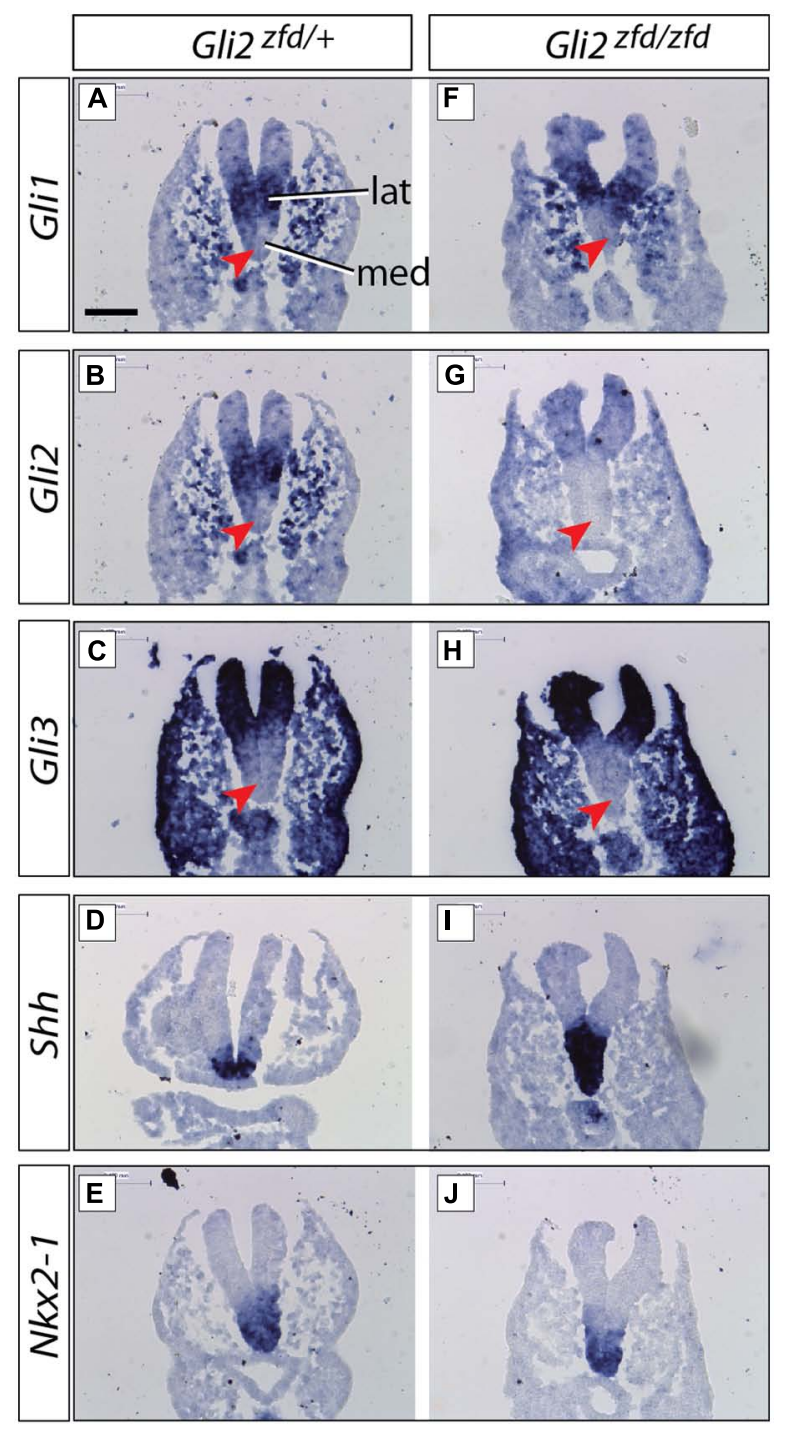

E8.5

FIGURE 2 | Expression of Gli genes in the presumptive hypothalamus at E8.5. In situ detection of marker gene expression in Glii2 zfd/+ and Gli2zfd/zfd mutant E8.5 embryos as indicated. "lat" and "med" in (A) indicate progenitor domains. Red arrowheads in (A-C,F-H) indicate lack of expression in the medial progenitor domain. $N k \times 2-1$ expression $(\mathbf{E}, \mathbf{J})$ identifies the presumptive hypothalamus. Scale bar (A) $100 \mu \mathrm{m}$.

alar hypothalamus have only a moderate phenotype, mostly evident in their reduced size (Szabo et al., 2009a; Zhao et al., 2012), indicating that they are specified by Shh of non-neural origin (e.g., from the prechordal plate or the notochord). Here we asked what is the role of Gli factors in those two hypothalamic regions by analyzing mutants in which Gli2, Gli3 or both neural Shh and Gli3 were inactivated. Expression of transcription factor Nkx2-1, an early preoptic marker (Shimamura et al., 1995; Xu et al., 2008), was preserved in the Gli2 $2^{z f d / z f d}$ and Gli3 ${ }^{X t-J / X t-J}$ mutants (black arrowheads in Figures 4A-C).Incidentally, some non-preoptic telencephalic expression domains were missing in the mutants (white arrowheads in Figures 4A-D). Arginin-Vasopressin (Avp) is specifically expressed by the supraoptic and paraventricular nuclei (Swanson and Sawchenko, 1983) and shows robust expression in both mutants (Figures $\mathbf{4 E}-\mathbf{G}$ ). The transcription factor gene $L h x 1$ is a marker of the suprachiasmatic nucleus (Szabo et al., 2009a), and this pattern remains essentially unchanged in the mutants (Figures 4I-K). Finally, analysis of double Foxb1Cre;Shht/f ;Gli3 ${ }^{X t-J / X t-J}$ mutants (lacking both neural Shh and Gli3) showed robust marker expression (Figures 4D,H,L).

These results could indicate that, downstream of Shh of nonneural origin at the early stage, Gli2 and Gli3 can fully substitute for each other's activator function in the alar portions of the hypothalamus or, alternatively, that the specification of the alar hypothalamus depends on suppression of Gli3R by non-neural Shh (Rallu et al., 2002).

\section{Gli2 is Required for the Development of Medial Tuberal and Mamillary Regions}

In order to analyze the Gli2 ${ }^{z f d / z f d}$ phenotype in the basal hypothalamus (tuberal and mamillary regions), we examined expression of Shh and Gli genes as well as regional markers at E8.5 and E10.5. At E8.5, expression of Gli1, Gli3 and the regional marker Nkx21 was not changed in the Gli2 $2^{z f d / z f d}$ mutant (Figures 2F,H-J), except of course for the disappearance of the Gli2 domain (Figure 2G). Expression of Shh, however, seemed expanded (Figure 2I). At E10.5, expression of Gli1 and Gli3 was strongly downregulated in the midline around the infundibular area (red arrowheads in Figures 3D,E,P,Q) in the Gli2 ${ }^{z f d / z f d}$ mutants. At mamillary levels, however, the two lateral expression domains seem to have fused in a thickened midline [Figures $3 \mathbf{F}, \mathbf{R}$; this is also true of the expression of the truncated (inactive) form of Gli2 in the mutant (Figure 3L)]. At this age, Shh expression is normally downregulated in the medial domain of the tuberal region (Manning et al., 2006; arrow in Figure 5A). In the Gli2 ${ }^{z f d / z f d}$ mutant this Shh-negative domain was absent (arrow in Figure 5B). $N k x 2-1$, a transcription factor gene defining regional specification of the basal hypothalamus (Kimura et al., 1996; Puelles et al., 2004, 2012), was expressed in an appropriate but smaller domain, with stronger expression shifted into the medial domain (Figures 5C,D). Six3 is a transcription factor required for initiation of hypothalamic specification (Kobayashi et al., 2002). It is normally expressed strongly along the entire medial domain and flanking hypothalamus, except the mamillary part. Six3 expression was severely reduced at both the infundibular (Figures 5E,F) and median eminence levels (Figures 5G,H). Together with the alterations in gene expression, we observed again a thickening of the medial domain of the tuberal region (arrowheads in Figures 5F,H). Analysis of Six3 expression on sagittal sections at E12.5 (Figures 5I,J) confirmed Six3 downregulation and a thickened medial domain of the Gli2 $2^{z f d / z f d}$ mutant (arrowheads in Figures 5I,J). Since expression of Six3 (Figures 5E,F) indicated alterations of the infundibulum, which is essential for pituitary development, we then examined the expression of appropriate gene markers for this region (Figure 6). Infundibular expression of Tbx2 (Manning et al., 2006) and Fgf8 (Ericson et al., 1998; Figures 6A-D), as well as expression of pituitary markers Lhx3 (Figures 6E,F), and Pitx2 (Figures 6 G,H) 

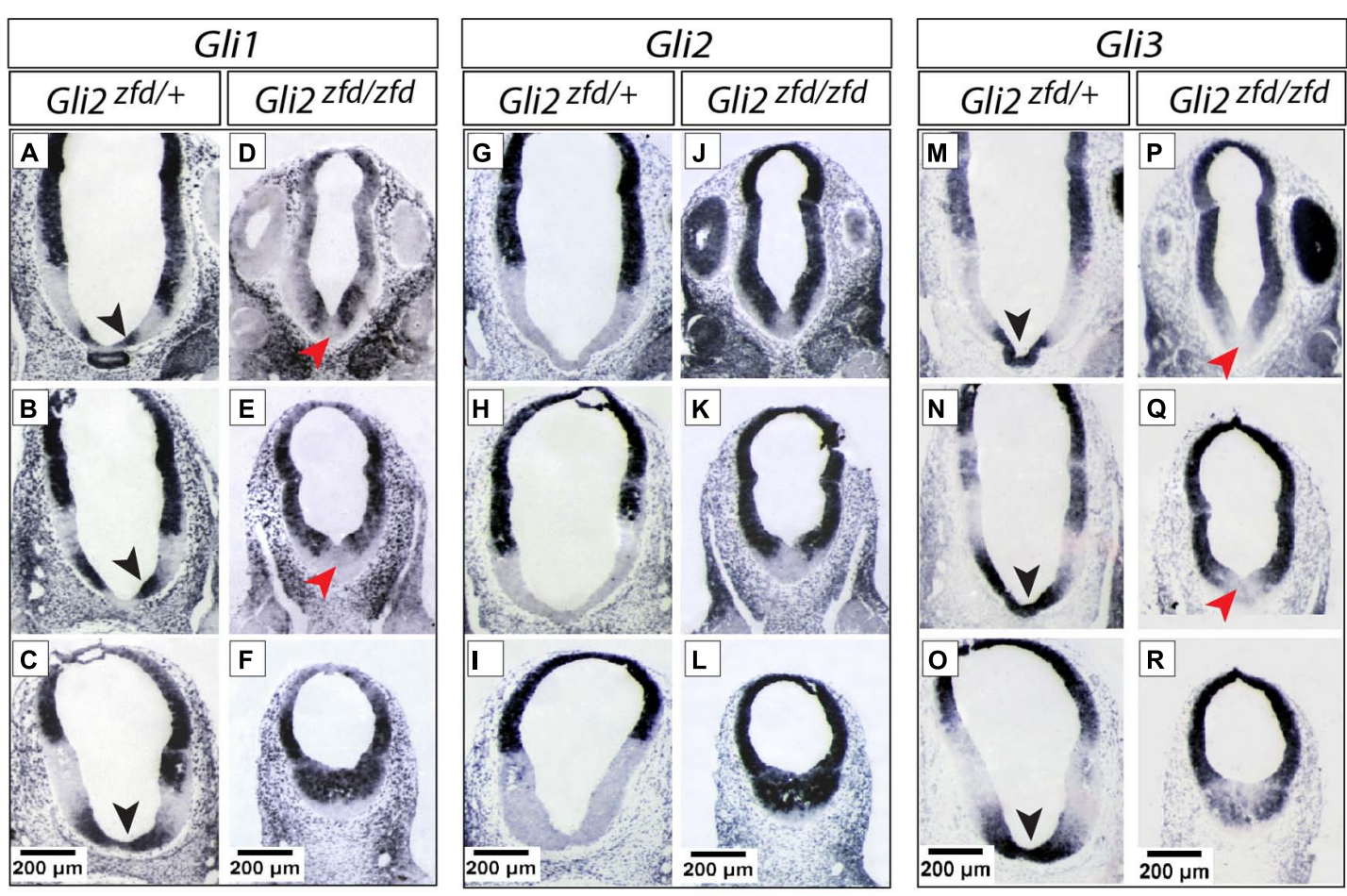

FIGURE 3 | Expression of Gli genes in the presumptive hypothalamus at E10.5. (A-R) In situ detection of Gli genes in the presumptive hypothalamus of E10.5 Gli2 zfd/+ and Gli2 zfd/zfd mouse embryos as indicated. For each gene, three levels are shown, from pituitary/infundibulum (top row) through mamillary region (bottom row). Black arrowheads in (A-C,M-O) show co-expression of Gli1 and Gli3 in the midline; red arrowheads in $(\mathbf{D}, \mathbf{E}, \mathbf{P}, \mathbf{Q})$ show downregulation of Gli1 and Gli3 in the Gli2 zfd/zfd midline. Scale bars, $200 \mu \mathrm{m}$. was completely lost in the Gli2 $2^{z f d} / z f d$ presumptive hypothalamus at E10.5 (see also Park et al., 2000). These results indicated that Gli2 is required for appropriate development of the medial domain in the basal hypothalamus and for the development of the neurohypophysis.

\section{Arcuate, Ventromedial, and Mamillary Nuclei are Severely Reduced in Size in the Gli2 ${ }^{\text {zfd/zfd }}$ Mutant}

We next analyzed the differentiation of the tuberal and mamillary regions in Gli2 ${ }^{z f d / z f d}$ brains at E18.5 (at this stage, characteristic neuronal nuclei are recognizable in the wildtype). $N p y$-expressing and Pomc-expressing neurons are specifically present in the arcuate nucleus (tuberal region; Elias et al., 1998; Figures 7A,C). In the Gli2 ${ }^{z f d / z f d}$ brain, the arcuate nuclei were not preserved as two distinct left and right domains. Instead, one single specifically labeled area was observed, unpaired and medial, sitting in the midline at the level of the tuberal area (dashed circle in Figures $\mathbf{7 B}, \mathbf{D}$ ). The third ventricle was abnormally absent at the site of this unpaired structure. Expression of SF-1 (nuclear receptor Nr5a1) specifically labels the ventromedial nucleus of the hypothalamus (Ikeda et al., 1995; Figure 7E). In the Gli2 $2^{z f d / z f d}$ brain, $S F-1$ was expressed in a median, unpaired group of cells (dashed circle in Figure 7F). The transcription factor $N k x 2-1$ is specifically expressed in the lateral part of the wildtype ventromedial nucleus (Nakamura et al., 2001; Figure 7G), but formed one single medial domain in the Gli2 ${ }^{z f d / z f d}$ brain (Figure $\mathbf{7 H}$ ). The transcription factor genes $\operatorname{Lhx} 1$, Otp, and Sim 1 are specifically expressed in the mamillary body (mamillary region) in the wildtype (Szabo et al., 2009a) but this expression was completely lost in the Gli2 ${ }^{z f d / z f d}$ mutant (Figures 7I-N). Together with the observations shown in Figures $\mathbf{5}$ and $\mathbf{6}$, these results indicate that Gli2 is essential for the specification of the medial progenitor domain (Alvarez-Bolado et al., 2012) of the basal hypothalamus. The Gli2 $2^{z f d / z f d}$ mutant mice showed an altered latero-medial organization of the molecular pattern of the basal hypothalamus consistent with a loss of the medial markers (notably reduced Six3 and loss of Tbx2, Otp, Sim1, and Lhx1) and derivatives (median eminence and neurohypophysis, mamillary body). The latter were substituted at the mutant midline by markers and derivatives typical of the lateral domain at this age, like $N k \times 2.1$, Npy, Pomc, and SF-1. That the neurohypophysis is a derivative from this region has been described before (Pearson et al., 2011; Pearson and Placzek, 2013).

\section{Gli2 in the Medial Progenitor Domain}

In the early presumptive hypothalamus (E7.5 to E8.5), an unpaired medial progenitor domain ("med" in Figures 2A and 11A) is specified, which gives rise to medially located nuclei like most of the arcuate nucleus, the medial portion of the ventromedial nucleus, the median eminence and the mamillary body (Alvarez-Bolado et al., 2012; derivatives of the acroterminal 

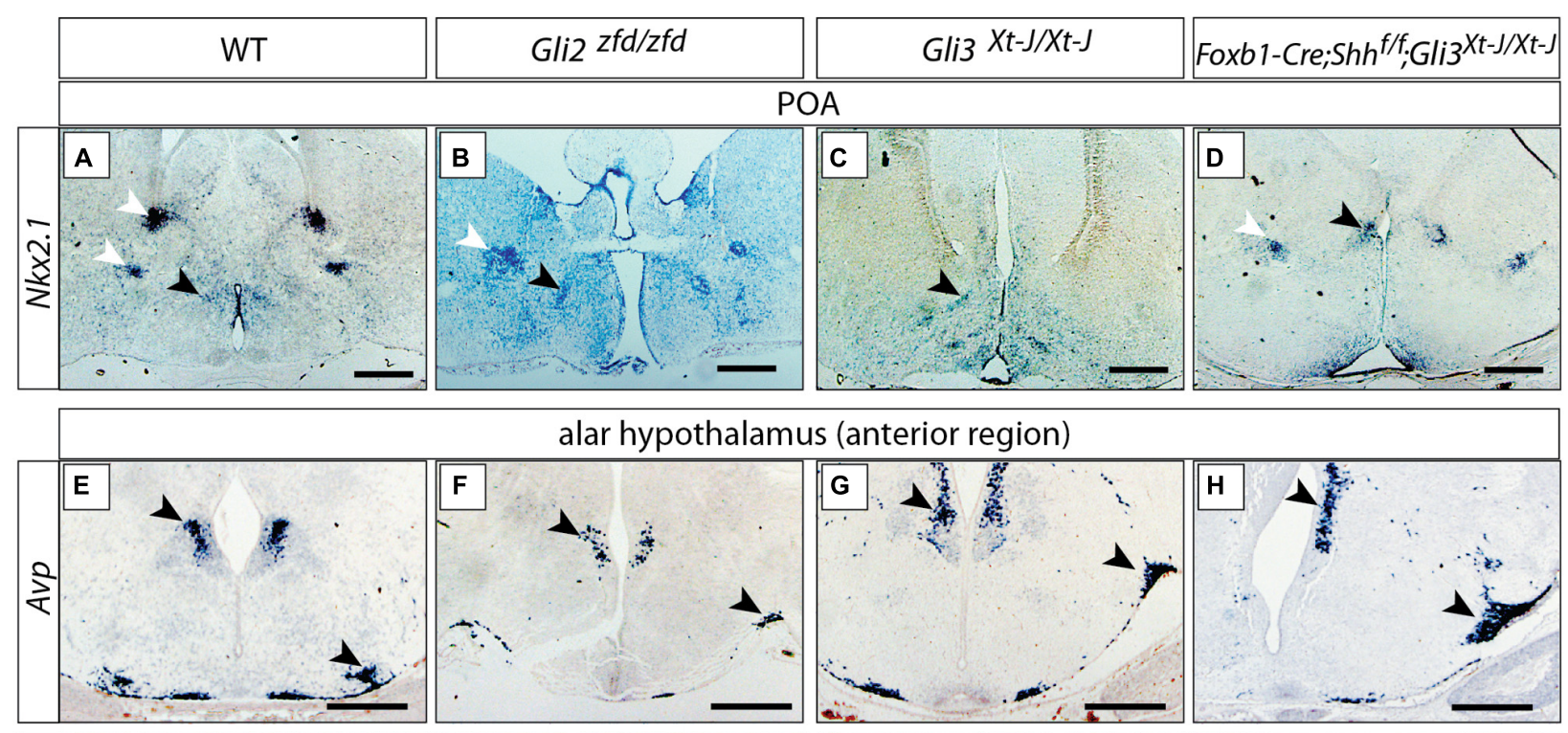

\section{alar hypothalamus (anterior region)}
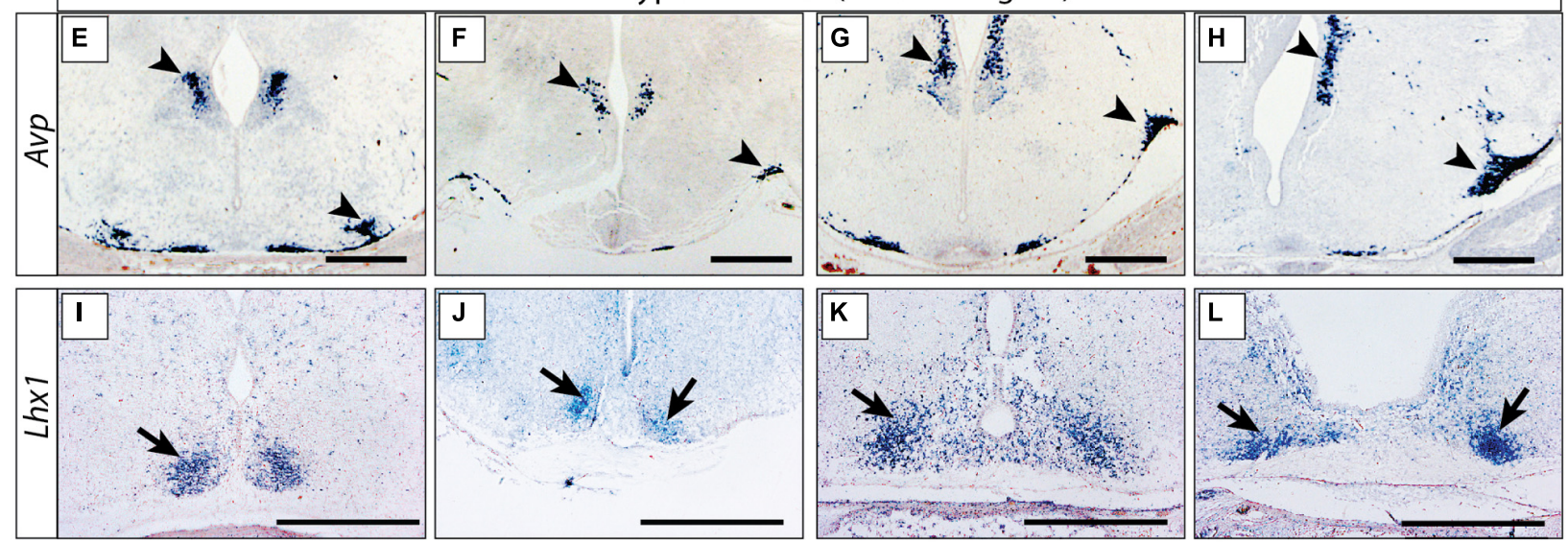

FIGURE 4 | Either Gli2A or Gli3A is sufficient for the specification of the preoptic region and alar hypothalamus. (A-D) In situ detection of preoptic marker gene Nkx2.1 on E18.5 mouse brain sections, genotypes as indicated.

telencephalic expression domains. (E-L) In situ detection of anterior marker genes on E18.5 mouse brain sections, markers and genotypes as indicated. Arrowheads in $\mathbf{E}-\mathbf{H}$ indicate the supraoptic and paraventricular nuclei; arrows in I-L indicate the suprachiasmatic nucleus. Scale bars $500 \mu \mathrm{m}$.

region, Puelles et al., 2012). This early domain and its lineage are strongly affected in the Gli2 $2^{z f d / z f d}$ mutant (Figures 5-7). Gli2 expression overlaps with Gli1 in the medial domain at E7.5 (Hui and Joyner, 1993). Since Gli1 expression is diagnostic of Shh pathway activation, this indicates a Gli2A function. The strong Gli2 $2^{z f d} / z f d$ midline phenotype must be due to a requirement for Gli2 expression in the medial domain at E7.5, since this domain does not show Gli2 expression at later stages (Figures 2 and 3). Moreover, expression of Gli1 and Gli3, normally absent from the midline at E8.5 (Figures 2A,C), is not ectopically upregulated in the Gli2 ${ }^{z f d / z f d}$ mutant (Figures 2F,H; i.e., no rescue). At E10.5, Gli3, and Gli1 expression overlap in the midline (black arrowheads in Figures $\mathbf{3 A - C}, \mathbf{M}-\mathbf{O}$ ) suggesting an activator role of Gli3 (Gli3A). However, both genes are strongly downregulated in the Gli2 $2^{z f d} / z f d$ midline at E10.5 (red arrowheads in Figures 3D,E,P,Q), again making a rescue of the Gli2 $2^{z f d} / z f d$ phenotype by a Gli3A function impossible.

\section{No Abnormal Phenotype in the Gli3 ${ }^{X t-J / X t-J}$ Basal Hypothalamus}

We went on to analyze the developing $G l i 3^{X t-J / X t-J}$ basal hypothalamus. At E18.5, expression of specific marker genes $N p y$, Pomc and SF-1 in the tuberal region (Figures 8A-F) and of $L h \times 1$ in the mamillary region (Figures $\mathbf{8} \mathbf{M}, \mathbf{N}$ ), showed that a Gli3A function in presence of Gli2A is dispensable for the specification of the basal hypothalamus.

We next addressed the question of a possible Gli3R function in the developing hypothalamus. Gli3R function often results in negative regulation of tissue growth (by inducing cell death and reducing proliferation) and counteracting the ventralizing influence of Shh (Persson et al., 2002; Ruiz i Altaba et al., 2003). Therefore, loss of Gli3R could result in ventralization and/or an increased size of hypothalamic nuclei. Since Shh signaling counteracts the processing of Gli3 protein into its repressor form, experimental abolition of Shh signaling might result in overabundance of Gli3R. We investigated this possibility by analyzing mouse mutants lacking Shh expression in the neural tube (Foxb1Cre;Shhf/f mutant, Szabo et al., 2009a). This mutant showed strong downregulation of three tuberal marker genes (Npy, Pomc, SF-1; Figures 8G-I) and had a more severe phenotype than the Gli2 ${ }^{z f d / z f d}$ mutants (Figures 7B,D,F,H; see also Szabo et al., 2009a; Shimogori et al., 2010). Given that Gli2A acts primarily at early stage of hypothalamic induction (see above) and following the logic of the Shh-Gli code (Bai et al., 2004), a possible explanation of this difference could be that, in the absence of neural Shh, formation of Gli3R is not prevented. A prediction of this hypothesis is that, in the absence of both neural Shh and Gli3 the 


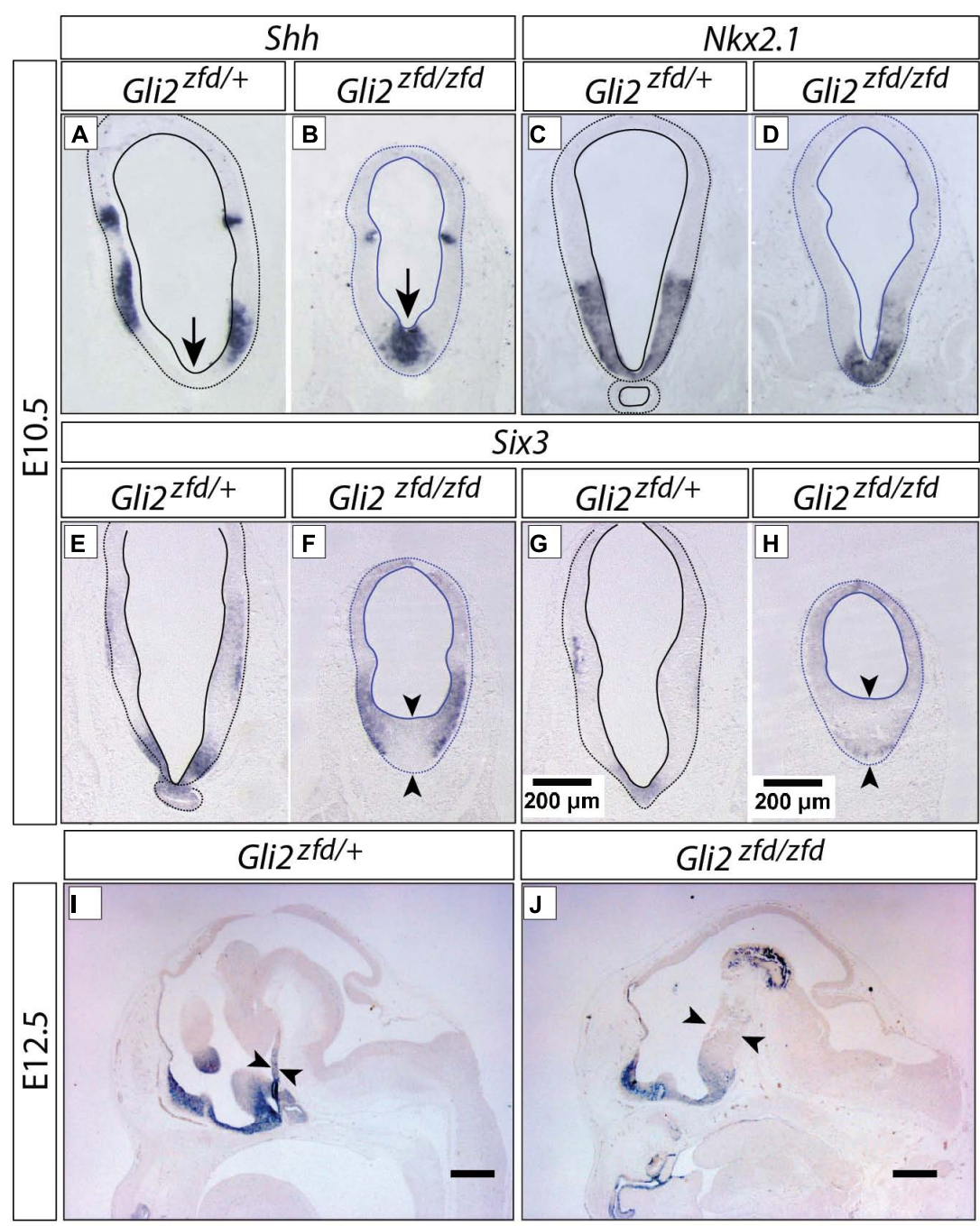

FIGURE 5|Abnormal medial domain in the Gli2 zfd/zfd mutant. (A-H) In situ detection of gene expression on forebrain sections of E10.5 embryos, genotypes, and markers as indicated. In $\mathbf{( E - H )}$, pituitary $\mathbf{( E , G )}$ and median eminence $\mathbf{( F , H )}$ levels are shown. Scale bar in (G,H), $200 \mu \mathrm{m} . \quad(\mathbf{I}, \mathbf{J})$ In situ detection of Six3 expression on sagittal sections of E12.5 WT (A) and Gli2 zfd/zfd (B) mouse embryos. Arrows show thickness of midline. Scale bars, $500 \mu \mathrm{m}$. tuberal region would have a less marked phenotype. We tested this prediction by analyzing mutants deficient not only in neural Shh but also in Gli3 (Foxb1-Cre; Shh ${ }^{f / f}$;Gli3 ${ }^{X t-J / X t-J}$ mutants). These showed essentially correct marker expression in the arcuate and ventromedial nuclei (although the expression domains appeared somewhat reduced and distorted; Figures 8J-L) suggesting that the phenotype in Foxb1-Cre;Shh $/ f$ mutants is at least partially caused by upregulated Gli3R activity. The same reasoning applies to the mamillary body (mamillary region), which is extremely reduced in the Foxb1-Cre;Shh ${ }^{f} f$ mutant (Szabo et al., 2009a) but appears normal in the Gli3 ${ }^{X t-J / X t-J}$ mutant and in Foxb1-Cre; Shh $/ f$; Gli3 ${ }^{X t-J / X t-J}$ double mutants (Figures 8M-O). We conclude that Gli3 is dispensable for overall hypothalamic specification. Moreover, it is likely that the upregulation of Gli3R is the main contributor to the defects in the tuberal and mamillary hypothalamus when neural $S h h$ is inactivated, which would be consistent with the classical Shh-Gli code in the spinal cord (Bai et al., 2004).

\section{A Possible Gli3 Activator Function in the Lateral Hypothalamic Area of the Gli2 zfd/zfd Mutant}

The LHA is a large and morphologically complex region and with key functions in the regulation of behavioral state and arousal mechanisms [reviewed in Swanson (2000)]. Analysis of Foxb1-Cre;Shhff mutants has shown that expression of Shh by the forebrain is essential for its specification (Szabo et al., 2009a). Hypocretin/orexin (Hcrt; Hungs and Mignot, 2001; Figures 9A-D) and pro-melanin-concentrating hormone (Pmch; Croizier et al., 2013; Figures 9E-H), essential modulators of metabolism and behavior, are among the very few specific marker genes of restricted groups of LHA neurons. The Gli3 $3^{X t-J / X t-J}$ 


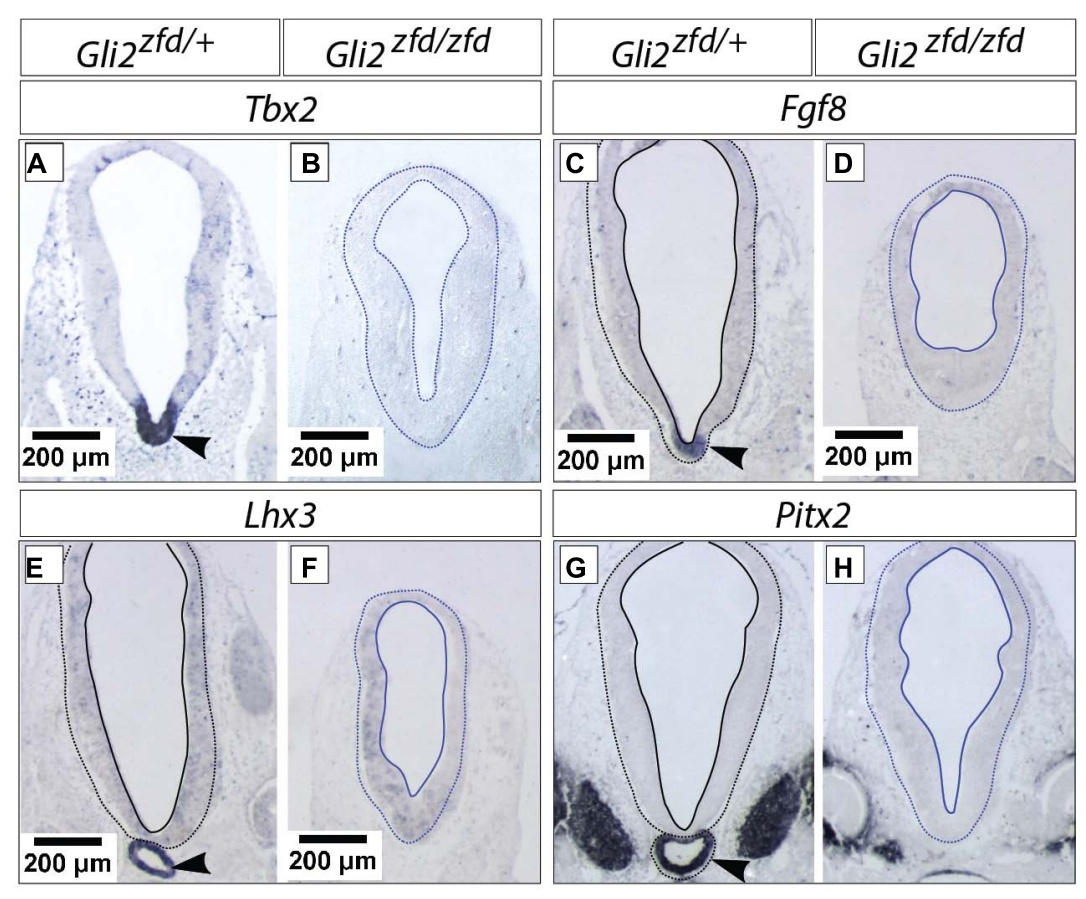

FIGURE 6 | Hypophysis region in the Gli2 zfd/zfd mutant at E10.5. In situ detection of infundibular and pituitary markers on Gli2 zfd/+ and Gli2 zfd/zfd E10.5 embryos as indicated. Arrowheads in (A,C,E,G) indicate normal expression domain. Scale bars, $200 \mu \mathrm{m}$.

brain did not show changes in Hcrt (Figure 9C) or Pmch expression (Figure 9G), indicating that Gli3A is normally not involved in the specification of the LHA. In the Gli2 $2^{z f d} / z f d$ mutant mice, only a few scattered Hcrt-expressing cells were present, and they were displaced toward the midline from their normal lateral position (arrowheads in Figure 9B). The number of Pmchexpressing neurons in the Gli2 ${ }^{z f d} / z f d$ mutant seemed not altered, but the cells tended to gather in the midline, similar to Hcrt cells (Figure 9F). This indicates that Gli2 is dispensable for the generation of Pmch-expressing cells, their altered position being rather a phenotypic consequence of the missing medial domain in this mutant (Figures 5 and 6). The phenotype of Foxb1-Cre;Shh $/ f$ mutants in this area (Hcrt cells are absent, and Pmch cells severely reduced Szabo et al., 2009a) is stronger that that of Gli2 $2^{z f d / z f d}$ mutants. We went on to address the possibility that a compensatory Gli3A function could explain the relatively mild LHA phenotype of Gli2 $2^{z f d / z f d}$ mutant mice. To test this hypothesis, we examined double mutants deficient in neural Shh and Gli3 (Foxb1-Cre;Shhf/f $; \mathrm{Gli3}^{\mathrm{Xt-J} / \mathrm{Xt-J}}$ ) and found a phenotype similar to that of the Foxb1-Cre;Shhf/f mutants (Hcrt cells absent, Pmch cells severely reduced; Figures 9D,H), but more pronounced than that of Gli2 $2^{z f d / z f d}$ mutants (Figures 9B,F). This indicates that, in the LHA of the Gli2 $2^{z f d / z f d}$ brain, Gli3A might compensate for the loss of Gli2A. This would be consistent with Gli1 still being expressed in the lateral domain of Gli2 ${ }^{z f d / z f d}$ mutants at E8.5 (Figure 2E).

We concluded that, for the specification of the LHA progenitors within the lateral progenitor domain, a Gli2A function is needed which can be partially substituted for by Gli3A.

\section{Gli3 in Mamillary Neurogenesis}

Differences in size can be due to quantitative changes in precursor generation (symmetric cell divisions) at an early stage or to later changes in neuron generation (asymmetric cell divisions). Shh is essential for the expansion of neural precursors in the early development of this region (Rowitch et al., 1999; Ishibashi and McMahon, 2002).

Here, we wanted to address the contribution of Shh-Gli to the neurogenesis of hypothalamic nuclei.

In situ analysis of Gli family expression at E12.5 (Figures 10A-C) showed Gli1 and Gli3 (arrowheads in Figures 10A,C) expression in the mamillary region. Gli2 expression on the contrary was very low or absent (arrowhead in Figure 10B). In agreement, a Gli3 reporter mouse line (Gli3$n L a c Z$ knock-in) showed strong beta-galactosidase labeling in the mamillary region (arrowhead in Figure 10D).

Therefore, we labeled proliferating cells in the neural tube by injecting BrdU in pregnant mice at E12.5 [i.e., at the peak of neurogenesis in the mouse hypothalamus (Ishii and Bouret, 2012)] and collecting the embryonic brains $3 \mathrm{~h}$ later. Our results (Figure 10E) show that proliferation during the neurogenic period in the mamillary region was reduced in the Gli3 ${ }^{X t-J / X t-J}$ mutant. Gli1 expression is diagnostic of Shh pathway activation, and can be found in the mamillary region at E10.5 (Figure 10F, upper panel), but it is lost in the Gli3 ${ }^{X t-J / X t-J}$ mutant (Figure 10F, lower panel), indicating failure of this domain to activate the Shh pathway in the mutant, in agreement with the reduced proliferation. BrdU labeling in this region was also reduced in the Gli2 $2^{z f d / z f d}$ mutant (Figure 10E), which we 


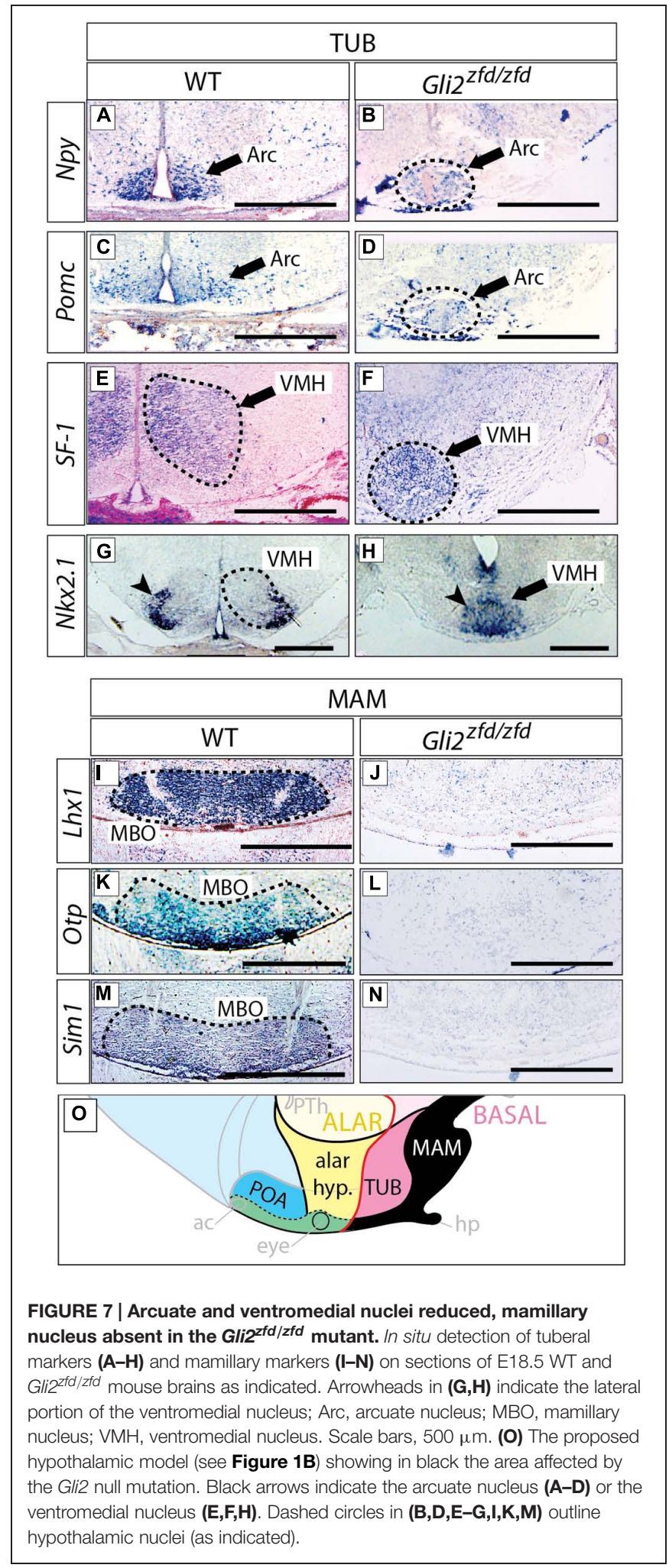

interpret as a consequence of the defect in midline development in these mutants (Figures 5 and 6). Intriguingly, deficiency in both neural Shh and Gli3 increased neurogenesis, particularly in the mamillary region (Figure 10E; see Discussion).
We then approached this issue experimentally by specifically blocking the Shh pathway in the hypothalamus of mouse embryos developing in utero. We electroporated wildtype embryos at E12.5 (Figures 10G-I) with EGFP-expressing reporter constructs mixed with constructs expressing the loss-of-function Shh receptor Ptch1- $\Delta$-loop2 plus the red fluorescent reporter tdTomato [similar to the one used by Briscoe et al. (2001); see Materials and Methods]. The results show that cells expressing high levels of Ptch1-delta-loop2 plus tdTomato in the mamillary region are less proliferative, while the same experiment did not alter neurogenesis in the alar hypothalamus (Figure 10J). We concluded that proliferation during the neurogenic period in the alar portion of the hypothalamus is not directly affected by Shh-Gli, while Gli3 has a role as an activator inducing neurogenesis at later stages in the basal regions.

\section{Discussion}

We asked which combinations of GliA and GliR specify different hypothalamic regions, and which members of the Gli family perform GliA or GliR functions. Therefore we interpreted mutant phenotypes with the help of Shh and Gli expression patterns (Figure 11A), hypothalamic progenitor domains (Alvarez-Bolado et al., 2012; Figure 11B), the Shh-Gli code established for the spinal cord (Bai et al., 2004) and a hypothalamic model (Figure 1B; Puelles et al., 2012). We uncovered strong differences in Gli gene requirements between alar and basal hypothalamus as predicted by the model. Null mutations of Gli2 or Gli3 do not alter the overall specification of the alar portions, including preoptic (actually telencephalic) and alar hypothalamus (or "anterior region"). In the basal regions (tuberal and mamillary), however, Gli2 is indispensable for the development of the medial progenitor domain and its derivatives but it is partly dispensable for the lateral progenitor domain (Figures 11B,D). Gli3 is dispensable for overall specification of the wildtype hypothalamus, but Gli3A has a late influence on mamillary proliferation. Finally, medial progenitor domain specification is dependent on Shh of non-neural source (prechordal plate, notochord), while the lateral progenitor domain is strongly dependent on neural Shh, in whose absence ectopic upregulation of Gli3R causes a severe phenotype. In this way, the notorious anatomical complexity of the hypothalamus depends on combinations of specification timing, progenitor domain, Shh source, Gli gene dependence, and alar vs. basal position (Figure 11D).

\section{Gli2A, Gli3A, and repression of Gli3R in hypothalamus specification}

Gli2 $2^{z f d / z f d}$ mutants lack a floor plate and its flanking cells from spinal cord to midbrain (Matise et al., 1998; Park et al., 2000). We extend this result to the rostral end of the floor plate (the mamillary region Puelles et al., 2012), and beyond this point, through the medial progenitor domain of the entire basal hypothalamus. Therefore, in the Gli2 $2^{z f d / z f d}$ mutant, the median eminence, pituitary and mamillary body are missing as well as part of the arcuate and ventromedial nuclei (Figure 11B). 


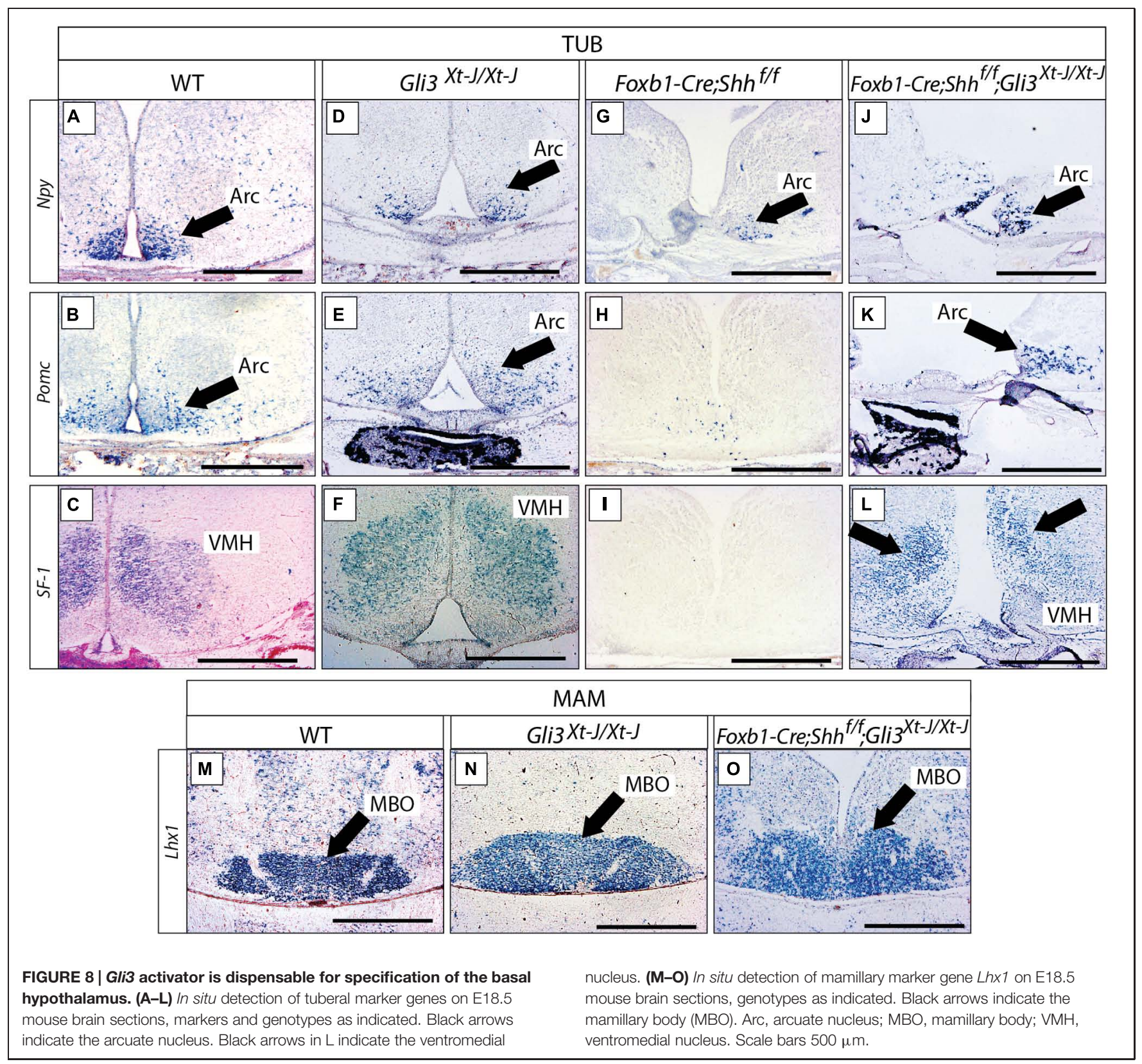

In spinal cord, Gli3 has important repressor (Gli3R; Litingtung and Chiang, 2000; Persson et al., 2002) and, in the absence of Gli2A, activator (Gli3A) functions (Bai et al., 2004). The lack of a pronounced phenotype in the Gli $3^{X t-J / X t-J}$ mutant hypothalamus indicates that under normal conditions either Gli3A is not important or that Gli2A can substitute for Gli3A. However, in the Gli2 $2^{z f d / z f d}$ mutant, the lateral progenitor domain is still able to produce part of the ventromedial and the arcuate nuclei and also the LHA (with only minor alteration; Figures $\mathbf{9 B}, \mathbf{F}$ ) - this can either be explained through rescue by a Gli3A function or it indicates that GliA is not essential for the induction of the lateral progenitor domain. A partial compensation through Gli3A (asterisk in Figure 11D) is supported by Gli1 expression in the lateral progenitor domain of the Gliz $2^{z f d / z f d}$ mutants (Figure 2F), where it partially overlaps with Gli3 (Figure $\mathbf{2 H}$ ). The suppression of Gli3R function by non-neural and neural Shh appears to be essential for the development of the hypothalamus (Figure 11D), since the phenotype of the ventral forebrain in Shh null mutants (Chiang et al., 1996) is more severe than in Gli2 $2^{z f d / z f d}$ mutants and the phenotype of the lateral progenitor domain in Foxb1Cre;Shh f $^{/ f}$ mutants (Szabo et al., 2009a) is more severe than in Gli2 $2^{z f d / z f d}$ mutants. In addition, the medial and lateral progenitor domains of Foxb1-Cre; Shht/f mutants are largely rescued in Foxb1-Cre;Shh $/ f ; G l i 3^{X t-J / X t-J}$ mice (Figures 8G-I,J-L). Finally, Hcrt+ neurons (part of the LHA) are reduced in Gli2 $2 f d / / f f d$ mutants and completely lost in Foxb1-Cre;Shhf/f mutants and even in Foxb1-Cre;Shh ${ }^{f / f}$; Gli3 ${ }^{X t-J / X t-J}$ brains, suggesting that their progenitors are uniquely specified by GliA or need GliA 


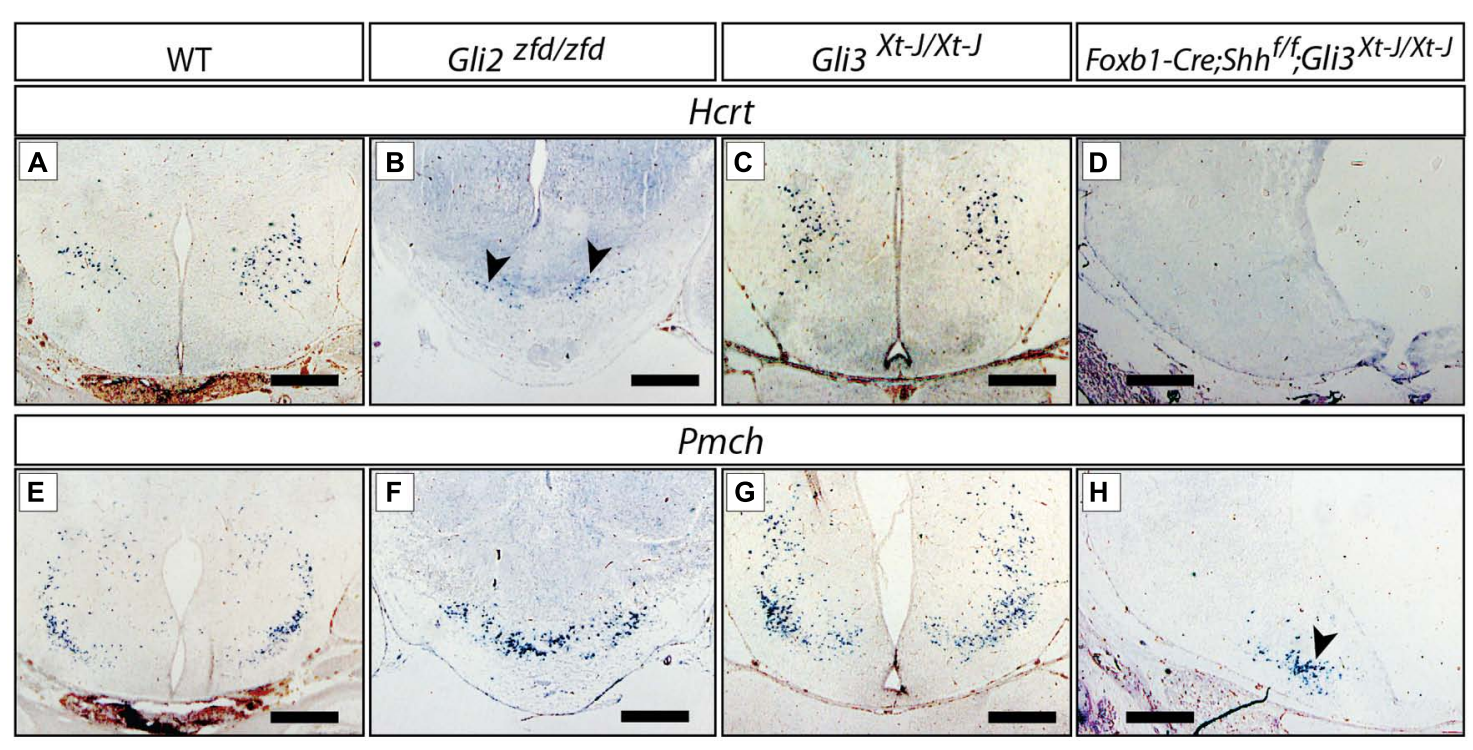

FIGURE 9 | Gli mutant phenotypes in the LHA. RNA in situ detection of lateral hypothalamic markers Hcrt/Orexin and Pmch on E18.5 mouse brain sections, genotypes as indicated. Arrowheads in $\mathbf{( B , H )}$, point at smaller groups of cells. Scale bars, $500 \mu \mathrm{m}$.

later or for a longer time than other progenitors in the lateral domain (Figure 11D). This is consistent with the very restricted place and time of neurogenesis of Hcrt+ neurons (Amiot et al., 2005). As mentioned in Section "Materials and Methods," we have not been able to obtain double mutant Gli2Gli3 embryos.

As for the alar hypothalamus and preoptic area, they are strongly dependent on Shh for their development, while Gli2 $2^{z f d / z f d}$ or Gli3 ${ }^{X t-J / X t-J}$ show no - or only subtle - phenotypes in these areas. This indicates that either Gli2A and Gli3A can fully substitute for each other in these regions, or they depend on suppression of Gli3R rather than induction of GliA by Shh for their specification (Chiang et al., 1996; Rallu et al., 2002).

\section{The Hypothalamic Version of the Shh-Gli Code}

In the spinal cord (Figure 11C), signaling by notochordal Shh is sufficient to generate the proper pattern of ventral progenitor gene expression (Matise et al., 1998; Jeong and McMahon, 2005), whereas ongoing Shh signaling from the floor plate (neural $S h h$ ) is necessary to maintain progenitor domain formation during neurogenesis (Dessaud et al., 2010) and for oligodendrocyte specification (Yu et al., 2013). We show that Shh of non-neural origin specifies the medial progenitor domain through Gli2A at an early stage, while neural Shh specifies the lateral progenitor domain at a later stage, probably by counteracting ectopic GliR function and, in the case of LHA progenitors, by inducing Gli2A (see above).

In both spinal cord (Bai et al., 2004; Figure 11C) and hypothalamus (Figures 11B,D), Gli2 performs the main GliA function. Gli2, however, is required for the induction of Shh expression in the floor plate (Matise et al., 1998), but not in the hypothalamus (Figure 2I).

Opposite gradients of GliA and GliR underlie the precise dorsoventral polarity of the spinal cord (Litingtung and Chiang, 2000; Persson et al., 2002) and hypothalamic specification requires counteracting Gli3R by Shh. Additionally, in the chicken hypothalamus, Gli3R activity is involved in $\operatorname{Pax} 7$ de-repression in some progenitors (Ohyama et al., 2008).

Finally, a Gli3A function is required for mamillary proliferation during the neurogenic phase (Figure 10E). The mamillary region overgrowth in Foxb1-Cre;Shht/f $; \mathrm{Gli}^{\mathrm{Xt}-J / X t-J}$ mutants (Figure 10E) parallels the abnormally increased size of the spinal cord in Gli2 ${ }^{z f d / z f d} ; G l i 3^{X t-J / X t-J}$ mutants. It remains unclear why inactivation of all Shh signaling results in a proliferation increase (Bai et al., 2004).

\section{Acroterminal Region vs. Floor Plate}

We have mapped the Gli2 $2^{z f d} /$ zfd phenotype (Figure 70) on a genetic-molecular model of the developing hypothalamus (Puelles et al., 2012) in which the ventral and dorsal midlines do not meet at a hypothetical "tip" of the neural tube (Figure 1B). Rather, the model proposes that the rostral end of the tube is closed by a "lid" in the form of a transverse structure called acroterminal region, which does not share the typical characteristics of the floor plate-e.g., it does not express Foxa2 (Ruiz i Altaba et al., 1995; Dale et al., 1999), undergoes complex, specific regulation (Ohyama et al., 2005; Manning et al., 2006; Ohyama et al., 2008; Trowe et al., 2013) and, as we show here, it is strongly neurogenic, not a property of the floor plate (except in the midbrain Kittappa et al., 2007; Ono et al., 2007; Bonilla et al., 2008). The histologically recognizable floor plate expresses Shh, Ntn1, Lmxb1, Foxa1, and Nr4a2 (Allen-Institute-for-Brain-Science, 2009; Puelles et al., 2012), is 

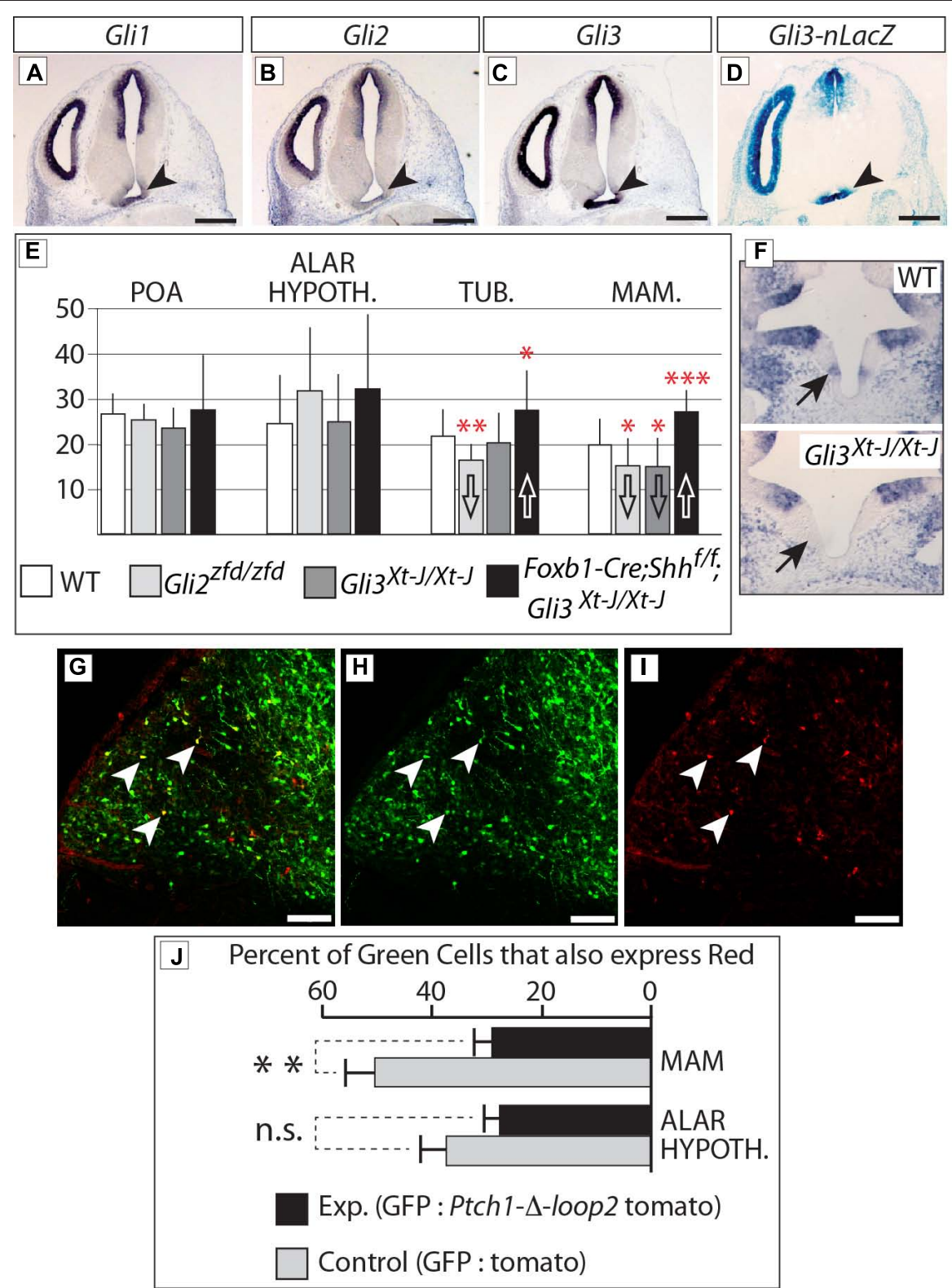

FIGURE 10 | Gli3 promotes proliferation in the mamillary region. (A-C) In situ detection of Gli genes on E12.5 WT mouse brain sections. Arrowheads indicate the mamillary region. (D) LacZ reporter detection on an E12.5 Gli3-nlacZ knock-in mouse brain section; arrowhead indicates the mamillary region. (E) BrdU-labeled cells per bin at E12.5, genotypes as indicated. Unpaired $t$-test, two-tailed, mean $\pm \mathrm{SD}$; ${ }^{*} p \leq 0.05$,

${ }^{* *} p \leq 0.01,{ }^{* * *} p \leq 0.001$. (F) Gli1 expression in the medial domain of the hypothalamus (arrows) on E10.5 horizontal sections of WT (upper panel) and Gli3 ${ }^{X t-J / X t-J}$ (lower panel) embryos. (G-I) Labeled cells in the mamillary region of WT E18.5 embryos after in utero electroporation with GFP and Ptch- $\Delta$-loop-tomato DNA constructs at E12.5. White arrowheads show double-labeled cells. (J) Percent of GFP-expressing cells co-expressing red reporter "tomato" after in utero electroporation of control (white bars) or experimental (black bars) constructs, in two different regions, as indicated. Unpaired $t$-test, two-tailed, mean $\pm \mathrm{SD}$; ${ }^{* *} p \leq 0.01 ;$ n.s., non-significant. induced by the underlying notochord, and it ends rostrally at mammillary level (Puelles et al., 2012). Beyond mammillary level, the acroterminal region extends all the way through the tuberal region, alar hypothalamus and preoptic region and up to the anterior commissure, it is transversally oriented (has alar and basal portions) and strongly patterned (probably by the underlying prechordal plate) and generates, among other, the median eminence, infundibulum, neurophypophysis, and eyes.

The dorso-ventral and rostro-caudal axes of the embryonic neural tube, considered in this way, are at a $90^{\circ}$ angle with those of the adult brain as they are usually considered; i.e., the adult rostro-caudal axis would be the dorso-ventral axis in our model. If this discrepancy will eventually be corrected remains 

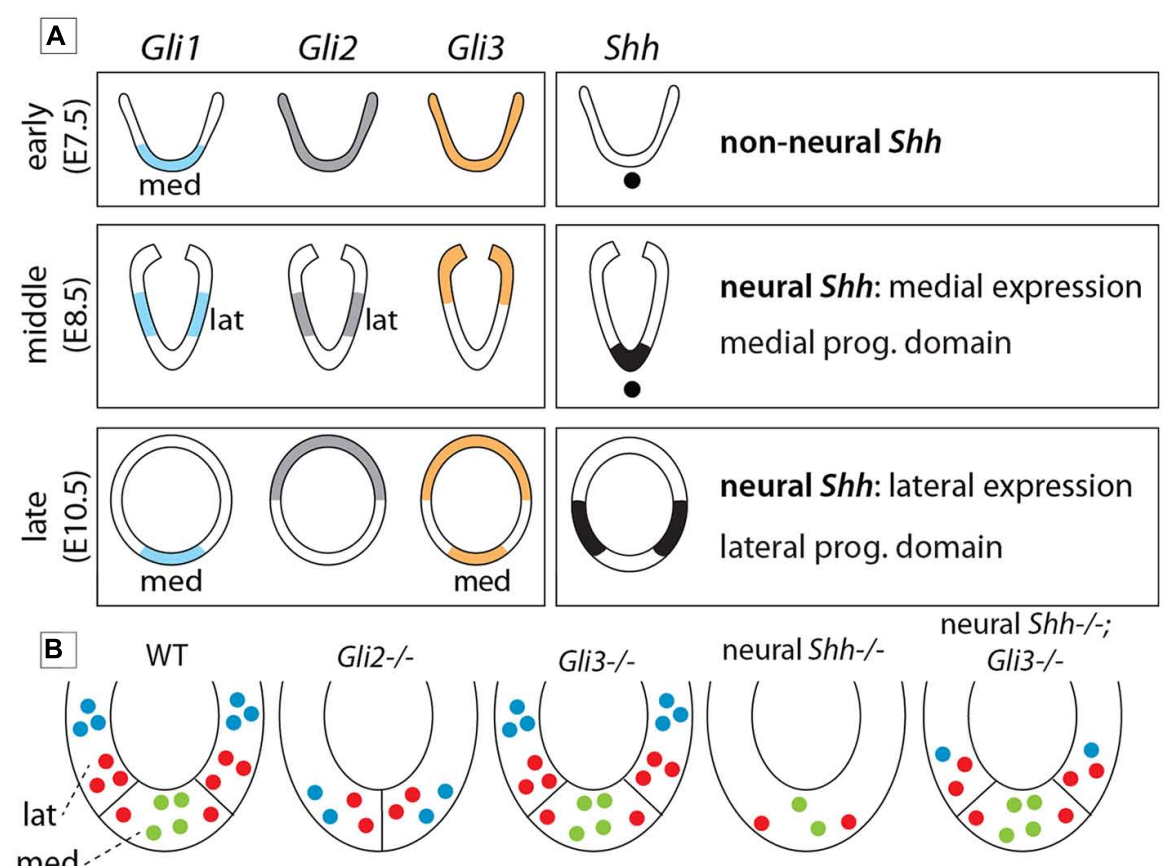

med -

- progenitors giving rise to midline structures o progenitors giving rise to LHA (median eminence, etc.) • progenitors giving rise to Arc and VMH
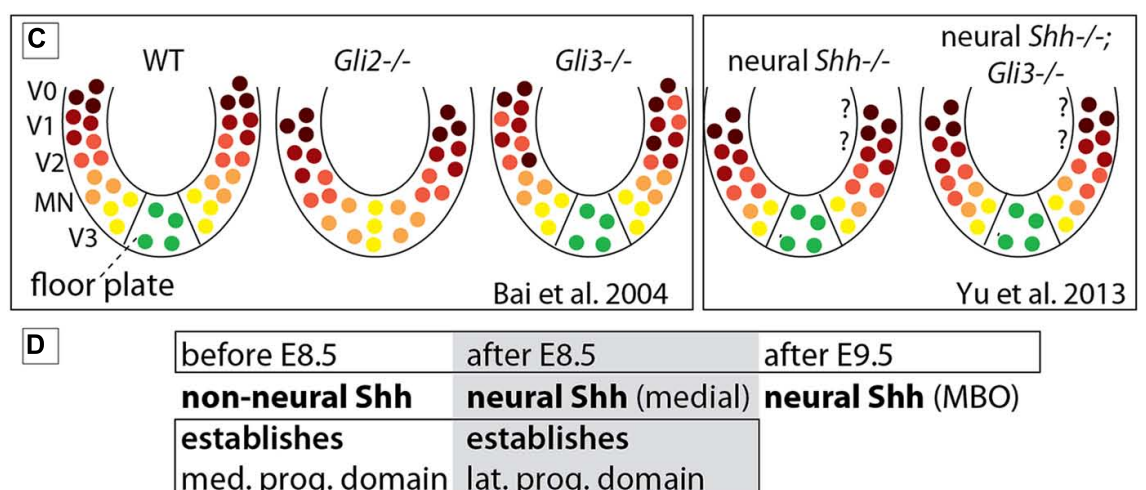

med. prog. domain lat. prog. domain

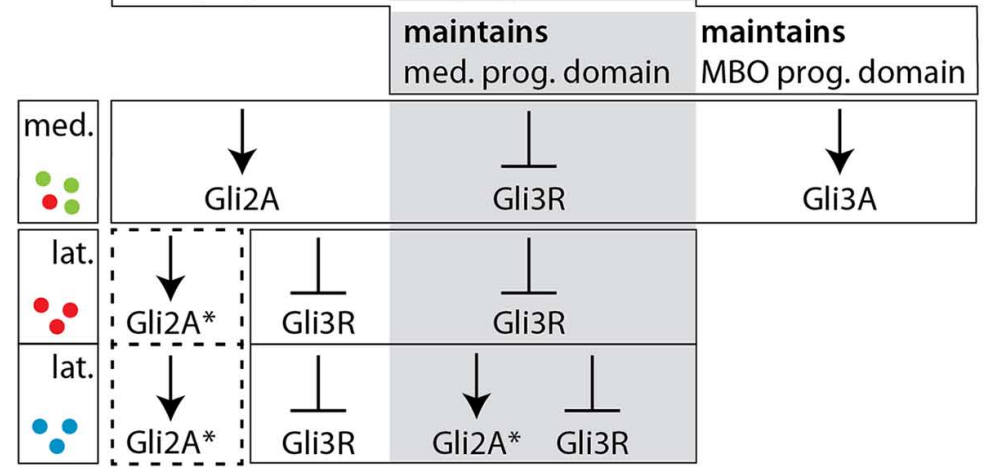

FIGURE 11| The role of each Gli protein in the basal hypothalamus of the mouse. (A) Diagram showing expression domains of the Gli factors and Shh in the presumptive hypothalamus at the early, middle, and late phases. "lat" and "med," lateral and medial domains, respectively (early phase according to Hui et al., 1994). (B) Summary diagrams of progenitor domains (neuroepithelium) of the basal hypothalamus in WT and mutants as deduced from phenotype analysis in the present study. (C) Diagrams comparable to those in $\mathbf{( B )}$ representing the progenitor domains in the spinal cord of WT and mutants as reported in the literature (as indicated). Question marks indicate that the V0/N1 domains have not been investigated. (D) Specific contribution of Gli proteins to the specification of the medial and lateral progenitor domains in three successive stages of development. Dotted square, possible influence of Gli2A on lateral progenitors before E8.5. The asterisk $\left(^{*}\right)$ means that loss of GliA2 could be compensated by Gli3A. MBO, mamillary body. See Discussion for details. 
open. The connectivity and function of the classical regions of the hypothalamus and the behavioral control column (Swanson, 2000) are not otherwise challenged by the proposed nomenclature (Puelles et al., 2012).

\section{The Gli2 zfd/zfd Phenotype and the Hypothalamic Model}

The Gli2 $2^{z f d / z f d}$ hypothalamic phenotype can be cleanly mapped (Figure 70) on the model of the embryonic hypothalamus (Puelles et al., 2012), which in turn receives experimental confirmation from our work. The basal regions depend specifically on Gli2. The alar hypothalamus and preoptic region, on the contrary, are not strictly dependent on Gli2A or Gli3A and are therefore genetically different. In this way, the basal/alar boundary, one main insight of the model, is confirmed. The basal part has unique genetic requirements, as much in the floor plate as in the acroterminal region, which are difficult to reconcile with a conventional rostral-caudal hypothalamic orientation (Figure 1A). Moreover, the medial and lateral progenitor domains of the basal hypothalamus (Alvarez-Bolado et al., 2012) can be mapped on the

\section{References}

Aberger, F., and Ruiz, I. A. A. (2014). Context-dependent signal integration by the GLI code: the oncogenic load, pathways, modifiers and implications for cancer therapy. Semin. Cell Dev. Biol. 33, 93-104. doi: 10.1016/j.semcdb.2014. 05.003

Allen-Institute-for-Brain-Science. (2009). Allen Developing Mouse Brain Atlas. Available at: http://developingmouse.brain-map.org

Alvarez-Bolado, G., Paul, F. A., and Blaess, S. (2012). Sonic hedgehog lineage in the mouse hypothalamus: from progenitor domains to hypothalamic regions. Neural Dev. 7, 4. doi: 10.1186/1749-8104-7-4

Amiot, C., Brischoux, F., Colard, C., La Roche, A., Fellmann, D., and Risold, P. Y. (2005). Hypocretin/orexin-containing neurons are produced in one sharp peak in the developing ventral diencephalon. Eur. J. Neurosci. 22, 531-534. doi: 10.1111/j.1460-9568.2005.04224.x

Aoto, K., Nishimura, T., Eto, K., and Motoyama, J. (2002). Mouse GLI3 regulates Fgf8 expression and apoptosis in the developing neural tube, face, and limb bud. Dev. Biol. 251, 320-332. doi: 10.1006/dbio.2002.0811

Bai, C. B., Auerbach, W., Lee, J. S., Stephen, D., and Joyner, A. L. (2002). Gli2, but not Gli1, is required for initial Shh signaling and ectopic activation of the Shh pathway. Development 129, 4753-4761.

Bai, C. B., Stephen, D., and Joyner, A. L. (2004). All mouse ventral spinal cord patterning by hedgehog is Gli dependent and involves an activator function of Gli3. Dev. Cell 6, 103-115. doi: 10.1016/S1534-5807(03)00394-0

Blaess, S., Bodea, G. O., Kabanova, A., Chanet, S., Mugniery, E., and Derouiche, A., et al. (2011). Temporal-spatial changes in Sonic hedgehog expression and signaling reveal different potentials of ventral mesencephalic progenitors to populate distinct ventral midbrain nuclei. Neural Dev. 6, 29. doi: 10.1186/17498104-6-29

Blaess, S., Corrales, J. D., and Joyner, A. L. (2006). Sonic hedgehog regulates Gli activator and repressor functions with spatial and temporal precision in the $\mathrm{mid} /$ hindbrain region. Development 133, 1799-1809. doi: 10.1242/dev.02339

Bonilla, S., Hall, A. C., Pinto, L., Attardo, A., Gotz, M., and Huttner, W. B., et al. (2008). Identification of midbrain floor plate radial glia-like cells as dopaminergic progenitors. Glia 56, 809-820. doi: 10.1002/glia.20654

Brewster, R., Lee, J., and Ruiz i Altaba, A. (1998). Gli/Zic factors pattern the neural plate by defining domains of cell differentiation. Nature 393, 579-583. doi: $10.1038 / 31242$

Briscoe, J., Chen, Y., Jessell, T. M., and Struhl, G. (2001). A hedgehog-insensitive form of patched provides evidence for direct long-range morphogen activity of sonic hedgehog in the neural tube. Mol. Cell 7, 1279-1291. doi: 10.1016/S10972765(01)00271-4 model too, corresponding to acroterminal and terminal hypothalamus (Puelles et al., 2012). Mapping other mutant phenotypes will refine the model and reveal fundamental aspects of brain development and organization. The patterning of the acroterminal region by the prechordal plate, for instance, is an open question.

\section{Acknowledgments}

This work was supported by the Deutsches Forschungsgemeinschaft Grant AL603/2-1 (to GAB); the North-Rhine-Westphalia Repatriation Program, Ministry for Innovation, Science and Research of North Rhine Westphalia (to SB); BFU2008-04156 (to LP); and Grant MR/K013750/1 from the Medical Research Council, MRC (to TT). RHT was the recipient of a DAAD Fellowship. We gratefully acknowledge the financial support of the Deutsche Forschungsgemeinschaft and Ruprecht-KarlsUniversität Heidelberg within the funding programme Open Access Publishing.

Caqueret, A., Yang, C., Duplan, S., Boucher, F., and Michaud, J. L. (2005). Looking for trouble: a search for developmental defects of the hypothalamus. Horm. Res. 64, 222-230. doi: 10.1159/000088977

Chiang, C., Litingtung, Y., Lee, E., Young, K. E., Corden, J. L., and Westphal, H., et al. (1996). Cyclopia and defective axial patterning in mice lacking Sonic hedgehog gene function. Nature 383, 407-413. doi: 10.1038/38 $3407 \mathrm{a} 0$

Croizier, S., Cardot, J., Brischoux, F., Fellmann, D., Griffond, B., and Risold, P. Y. (2013). The vertebrate diencephalic MCH system: a versatile neuronal population in an evolving brain. Front. Neuroendocrinol. 34:65-87. doi: 10.1016/j.yfrne.2012.10.001

Dale, K., Sattar, N., Heemskerk, J., Clarke, J. D., Placzek, M., and Dodd, J. (1999). Differential patterning of ventral midline cells by axial mesoderm is regulated by BMP7 and chordin. Development 126, 397-408.

Dassule, H. R., Lewis, P., Bei, M., Maas, R., and McMahon, A. P. (2000). Sonic hedgehog regulates growth and morphogenesis of the tooth. Development 127 , 4775-4785.

Dessaud, E., McMahon, A. P., and Briscoe, J. (2008). Pattern formation in the vertebrate neural tube: a sonic hedgehog morphogen-regulated transcriptional network. Development 135, 2489-2503. doi: 10.1242/dev.009324

Dessaud, E., Ribes, V., Balaskas, N., Yang, L. L., Pierani, A., and Kicheva, A., et al. (2010). Dynamic assignment and maintenance of positional identity in the ventral neural tube by the morphogen sonic hedgehog. PLoS Biol. 8:e1000382. doi: 10.1371/journal.pbio.1000382

Echelard, Y., Epstein, D. J., St-Jacques, B., Shen, L., Mohler, J., and McMahon, J. A., et al. (1993). Sonic hedgehog, a member of a family of putative signaling molecules, is implicated in the regulation of CNS polarity. Cell 75, 1417-1430. doi: 10.1016/0092-8674(93)90627-3

Elias, C. F., Saper, C. B., Maratos-Flier, E., Tritos, N. A., Lee, C., and Kelly, J., et al. (1998). Chemically defined projections linking the mediobasal hypothalamus and the lateral hypothalamic area. J. Comp. Neurol. 402, 442-459. doi: 10.1002/(SICI)1096-9861(19981228)402:4<442::AID-CNE2>3.0.CO;2-R

Ericson, J., Briscoe, J., Rashbass, P., van Heyningen, V., and Jessell, T. M. (1997). Graded sonic hedgehog signaling and the specification of cell fate in the ventral neural tube. Cold Spring Harb. Symp. Quant. Biol. 62, 451-466. doi: 10.1101/SQB.1997.062.01.053

Ericson, J., Muhr, J., Placzek, M., Lints, T., Jessell, T. M., and Edlund, T. (1995). Sonic hedgehog induces the differentiation of ventral forebrain neurons: a common signal for ventral patterning within the neural tube. Cell 81, 747-756. doi: 10.1016/0092-8674(95)90536-7

Ericson, J., Norlin, S., Jessell, T. M., and Edlund, T. (1998). Integrated FGF and BMP signaling controls the progression of progenitor cell differentiation and 
the emergence of pattern in the embryonic anterior pituitary. Development 125 , 1005-1015.

Feijoo, C. G., Onate, M. G., Milla, L. A., and Palma, V. A. (2011). Sonic hedgehog (Shh)-Gli signaling controls neural progenitor cell division in the developing tectum in zebrafish. Eur. J. Neurosci. 33, 589-598. doi: 10.1111/j.14609568.2010.07560.x

Garcia, A. D., Petrova, R., Eng, L., and Joyner, A. L. (2010). Sonic hedgehog regulates discrete populations of astrocytes in the adult mouse forebrain. J. Neurosci. 30, 13597-13608. doi: 10.1523/JNEUROSCI.083010.2010

Genestine, M., Robert, B., and Lallemand, Y. (2007). High-resolution mapping of the Gli3 deletion in the mouse extra-toesH mutant. Genesis 45, 107-112. doi: 10.1002/dvg.20270

Haddad-Tovolli, R., Heide, M., Zhou, X., Blaess, S., and Alvarez-Bolado, G. (2012). Mouse thalamic differentiation: gli-dependent pattern and gliindependent prepattern. Front. Neurosci. 6:27. doi: 10.3389/fnins.2012. 00027

Haddad-Tovolli, R., Szabo, N. E., Zhou, X., and Alvarez-Bolado, G. (2013). Genetic manipulation of the mouse developing hypothalamus through in utero electroporation. J. Vis. Exp. 77. doi: 10.3791/50412

Hui, C. C., and Joyner, A. L. (1993). A mouse model of greig cephalopolysyndactyly syndrome: the extra-toesJ mutation contains an intragenic deletion of the Gli3 gene. Nat. Genet. 3, 241-246. doi: 10.1038/ng0393-241

Hui, C. C., Slusarski, D., Platt, K. A., Holmgren, R., and Joyner, A. L. (1994). Expression of three mouse homologs of the Drosophila segment polarity gene cubitus interruptus, Gli, Gli-2, and Gli-3, in ectoderm- and mesoderm-derived tissues suggests multiple roles during postimplantation development. Dev. Biol. 162, 402-413. doi: 10.1006/dbio.1994.1097

Hungs, M., and Mignot, E. (2001). Hypocretin/orexin, sleep and narcolepsy. Bioessays 23, 397-408. doi: 10.1002/bies.1058

Ikeda, Y., Luo, X., Abbud, R., Nilson, J. H., and Parker, K. L. (1995). The nuclear receptor steroidogenic factor 1 is essential for the formation of the ventromedial hypothalamic nucleus. Mol. Endocrinol. 9, 478-486.

Ingham, P. W., Nakano, Y., and Seger, C. (2011). Mechanisms and functions of Hedgehog signalling across the metazoa. Nat. Rev. Genet. 12, 393-406. doi: $10.1038 / \mathrm{nrg} 2984$

Ishibashi, M., and McMahon, A. P. (2002). A sonic hedgehog-dependent signaling relay regulates growth of diencephalic and mesencephalic primordia in the early mouse embryo. Development 129, 4807-4819.

Ishii, Y., and Bouret, S. G. (2012). Embryonic birthdate of hypothalamic leptin-activated neurons in mice. Endocrinology 153, 3657-3667. doi: 10.1210/en.2012-1328

Jeong, J., and McMahon, A. P. (2005). Growth and pattern of the mammalian neural tube are governed by partially overlapping feedback activities of the hedgehog antagonists patched 1 and Hhip1. Development 132, 143-154. doi: 10.1242/dev.01566

Kimura, S., Hara, Y., Pineau, T., Fernandez-Salguero, P., Fox, C. H., and Ward, J. M., et al. (1996). The T/ebp null mouse: thyroid-specific enhancerbinding protein is essential for the organogenesis of the thyroid, lung, ventral forebrain, and pituitary. Genes Dev. 10, 60-69. doi: 10.1101/gad. 10.1 .60

Kittappa, R., Chang, W. W., Awatramani, R. B., and McKay, R. D. (2007). The foxa2 gene controls the birth and spontaneous degeneration of dopamine neurons in old age. PLoS Biol. 5:e325. doi: 10.1371/journal.pbio.0050325

Kobayashi, D., Kobayashi, M., Matsumoto, K., Ogura, T., Nakafuku, M., and Shimamura, K. (2002). Early subdivisions in the neural plate define distinct competence for inductive signals. Development 129, 83-93.

Lebel, M., Mo, R., Shimamura, K., and Hui, C. C. (2007). Gli2 and Gli3 play distinct roles in the dorsoventral patterning of the mouse hindbrain. Dev. Biol. 302, 345-355. doi: 10.1016/j.ydbio.2006.08.005

Lee, J., Platt, K. A., Censullo, P., and Ruiz i Altaba, A. (1997). Gli1 is a target of Sonic hedgehog that induces ventral neural tube development. Development 124, 2537-2552.

Lewis, P. M., Dunn, M. P., McMahon, J. A., Logan, M., Martin, J. F., and St-Jacques, B., et al. (2001). Cholesterol modification of sonic hedgehog is required for long-range signaling activity and effective modulation of signaling by Ptc1. Cell 105, 599-612. doi: 10.1016/S0092-8674(01) 00369-5
Litingtung, Y., and Chiang, C. (2000). Specification of ventral neuron types is mediated by an antagonistic interaction between Shh and Gli3. Nat. Neurosci. 3, 979-985. doi: 10.1038/79916

Manning, L., Ohyama, K., Saeger, B., Hatano, O., Wilson, S. A., and Logan, M., et al. (2006). Regional morphogenesis in the hypothalamus: a BMP-Tbx2 pathway coordinates fate and proliferation through Shh downregulation. Dev. Cell 11, 873-885. doi: 10.1016/j.devcel.2006.09.021

Matise, M. P., Epstein, D. J., Park, H. L., Platt, K. A., and Joyner, A. L. (1998). Gli2 is required for induction of floor plate and adjacent cells, but not most ventral neurons in the mouse central nervous system. Development 125, 2759-2770.

Maynard, T. M., Jain, M. D., Balmer, C. W., and LaMantia, A. S. (2002). Highresolution mapping of the Gli3 mutation Extra-toes J reveals a 51.5-kb deletion. Mamm. Genome 13, 58-61. doi: 10.1007/s00335-001-2115-X

McMillen, I. C., MacLaughlin, S. M., Muhlhausler, B. S., Gentili, S., Duffield, J. L., and Morrison, J. L. (2008). Developmental origins of adult health and disease: the role of periconceptional and foetal nutrition. Basic Clin. Pharmacol. Toxicol. 102, 82-89. doi: 10.1111/j.1742-7843.2007.00188.x

Mo, R., Freer, A. M., Zinyk, D. L., Crackower, M. A., Michaud, J., and Heng, H. H., et al. (1997). Specific and redundant functions of Gli2 and Gli3 zinc finger genes in skeletal patterning and development. Development 124, 113-123.

Motoyama, J., Milenkovic, L., Iwama, M., Shikata, Y., Scott, M. P., and Hui, C. C. (2003). Differential requirement for Gli2 and Gli3 in ventral neural cell fate specification. Dev. Biol. 259, 150-161. doi: 10.1016/S0012-1606(03) 00159-3

Nakamura, K., Kimura, S., Yamazaki, M., Kawaguchi, A., Inoue, K., and Sakai, T. (2001). Immunohistochemical analyses of thyroid-specific enhancerbinding protein in the fetal and adult rat hypothalami and pituitary glands. Brain Res. Dev. Brain Res. 130, 159-166. doi: 10.1016/S0165-3806(01)00226-7

Niwa, H., Yamamura, K., and Miyazaki, J. (1991). Efficient selection for highexpression transfectants with a novel eukaryotic vector. Gene 108, 193-199. doi: 10.1016/0378-1119(91)90434-D

Ohyama, K., Das, R., and Placzek, M. (2008). Temporal progression of hypothalamic patterning by a dual action of BMP. Development 135, 3325-3331. doi: 10.1242/dev.027078

Ohyama, K., Ellis, P., Kimura, S., and Placzek, M. (2005). Directed differentiation of neural cells to hypothalamic dopaminergic neurons. Development 132 , 5185-5197. doi: 10.1242/dev.02094

Ono, Y., Nakatani, T., Sakamoto, Y., Mizuhara, E., Minaki, Y., and Kumai, M., et al. (2007). Differences in neurogenic potential in floor plate cells along an anteroposterior location: midbrain dopaminergic neurons originate from mesencephalic floor plate cells. Development 134, 3213-3225. doi: 10.1242/ dev.02879

Osorio, J., Mazan, S., and Retaux, S. (2005). Organisation of the lamprey (Lampetra fluviatilis) embryonic brain: Insights from LIM-homeodomain, Pax and Hedgehog genes. Dev. Biol. 288, 100-112. doi: 10.1016/j.ydbio.2005.08.042

Pabst, O., Herbrand, H., Takuma, N., and Arnold, H. H. (2000). NKX2 gene expression in neuroectoderm but not in mesendodermally derived structures depends on sonic hedgehog in mouse embryos. Dev. Genes Evol. 210, 47-50. doi: 10.1007/PL00008188

Park, H. L., Bai, C., Platt, K. A., Matise, M. P., Beeghly, A., Hui, C. C., et al. (2000). Mouse Gli1 mutants are viable but have defects in SHH signaling in combination with a Gli2 mutation. Development 127, 1593-1605.

Pavletich, N. P., and Pabo, C. O. (1993). Crystal structure of a five-finger GLIDNA complex: new perspectives on zinc fingers. Science 261, 1701-1707. doi: $10.1126 /$ science. 8378770

Pearson, C. A., Ohyama, K., Manning, L., Aghamohammadzadeh, S., Sang, H., and Placzek, M. (2011). FGF-dependent midline-derived progenitor cells in hypothalamic infundibular development. Development 138, 2613-2624. doi: 10.1242/dev.062794

Pearson, C. A., and Placzek, M. (2013). Development of the medial hypothalamus: forming a functional hypothalamic-neurohypophyseal interface. Curr. Top. Dev. Biol 106, 49-88. doi: 10.1016/B978-0-12-416021-7.00002-X

Persson, M., Stamataki, D., te Welscher, P., Andersson, E., Bose, J., and Ruther, U., et al. (2002). Dorsal-ventral patterning of the spinal cord requires Gli3 transcriptional repressor activity. Genes Dev. 16, 2865-2878. doi: $10.1101 / \operatorname{gad} .243402$

Puelles, L., Martínez, S., Martínez-de-la-Torre, M., and Rubenstein, J. L. R. (2004). "Gene maps and related histogenetic domains in the forebrain and midbrain," 
in The Rat Nervous System, ed. G. Paxinos (San Diego, CA: Academic Press), 3-25.

Puelles, L., Martinez-de-la-Torre, M., Bardet, S., and Rubenstein, J. L. R. (2012). "Hypothalamus," in The Mouse Nervous System, eds C. Watson, G. Paxinos, and L. Puelles (San Diego, CA: Elsevier-Academic Press), 221-312. doi: 10.1016/B978-0-12-369497-3.10008-1

Rallu, M., Machold, R., Gaiano, N., Corbin, J. G., McMahon, A. P., and Fishell, G. (2002). Dorsoventral patterning is established in the telencephalon of mutants lacking both Gli3 and Hedgehog signaling. Development 129, 4963-4974.

Rowitch, D. H., S-Jacques, B., Lee, S. M., Flax, J. D., Snyder, E. Y., and McMahon, A. P. (1999). Sonic hedgehog regulates proliferation and inhibits differentiation of CNS precursor cells. J. Neurosci. 19, 8954-8965.

Ruiz i Altaba, A. (1997). Catching a Gli-mpse of hedgehog. Cell 90, 193-196. doi: 10.1016/S0092-8674(00)80325-6

Ruiz i Altaba, A., Jessell, T. M., and Roelink, H. (1995). Restrictions to floor plate induction by hedgehog and winged-helix genes in the neural tube of frog embryos. Mol. Cell. Neurosci. 6, 106-121. doi: 10.1006/mcne.1995.1011

Ruiz i Altaba, A., Nguyen, V., and Palma, V. (2003). The emergent design of the neural tube: prepattern, SHH morphogen and GLI code. Curr. Opin. Genet. Dev. 13, 513-521. doi: 10.1016/j.gde.2003.08.005

Saito, T. (2006). In vivo electroporation in the embryonic mouse central nervous system. Nat. Protoc. 1, 1552-1558. doi: 10.1038/nprot.2006.276

Saito, T., and Nakatsuji, N. (2001). Efficient gene transfer into the embryonic mouse brain using in vivo electroporation. Dev. Biol. 240, 237-246. doi: 10.1006/dbio.2001.0439

Saper, C. B. (2006). Staying awake for dinner: hypothalamic integration of sleep, feeding, and circadian rhythms. Prog. Brain Res. 153, 243-252. doi: 10.1016/S0079-6123(06)53014-6

Shimamura, K., Hartigan, D. J., Martinez, S., Puelles, L., and Rubenstein, J. L. (1995). Longitudinal organization of the anterior neural plate and neural tube. Development 121, 3923-3933.

Shimogori, T., Lee, D. A., Miranda-Angulo, A., Yang, Y., Wang, H., and Jiang, L., et al. (2010). A genomic atlas of mouse hypothalamic development. Nat. Neurosci. 13, 767-775. doi: 10.1038/nn.2545

Sternson, S. M. (2013). Hypothalamic survival circuits: blueprints for purposive behaviors. Neuron 77, 810-824. doi: 10.1016/j.neuron.2013.02.018

Swanson, L. W. (1987). "The hypothalamus," in Handbook of Chemical Neuroanatomy, eds A. Björlund, T. Hökfelt, and L. W. Swanson, (Amsterdam: Elsevier), 1-124.

Swanson, L. W. (2000). Cerebral hemisphere regulation of motivated behavior. Brain Res. 886, 113-164. doi: 10.1016/S0006-8993(00) 02905-X

Swanson, L. W., and Sawchenko, P. E. (1983). Hypothalamic integration: organization of the paraventricular and supraoptic nuclei. Annu. Rev. Neurosci. 6, 269-324. doi: 10.1146/annurev.ne.06.030183.001413

Szabo, N. E., Zhao, T., Cankaya, M., Theil, T., Zhou, X., and Alvarez-Bolado, G. (2009a). Role of neuroepithelial Sonic hedgehog in hypothalamic patterning. J. Neurosci. 29, 6989-7002. doi: 10.1523/JNEUROSCI.1089-09.2009
Szabo, N. E., Zhao, T., Zhou, X., and Alvarez-Bolado, G. (2009b). The role of Sonic hedgehog of neural origin in thalamic differentiation in the mouse. J. Neurosci. 29, 2453-2466. doi: 10.1523/JNEUROSCI.4524-08.2009

Takahashi, T., Nowakowski, R. S., and Caviness, V. S. Jr. (1993). Cell cycle parameters and patterns of nuclear movement in the neocortical proliferative zone of the fetal mouse. J. Neurosci. 13, 820-833.

Trowe, M. O., Zhao, L., Weiss, A. C., Christoffels, V., Epstein, D. J., and Kispert, A. (2013). Inhibition of Sox2-dependent activation of Shh in the ventral diencephalon by $\mathrm{Tbx} 3$ is required for formation of the neurohypophysis. Development 140, 2299-2309. doi: 10.1242/dev.094524

Wang, M. Z., Jin, P., Bumcrot, D. A., Marigo, V., McMahon, A. P., and Wang, E. A., et al. (1995). Induction of dopaminergic neuron phenotype in the midbrain by Sonic hedgehog protein. Nat. Med. 1, 1184-1188. doi: 10.1038/nm1195-1184

Warren, N., Caric, D., Pratt, T., Clausen, J. A., Asavaritikrai, P., and Mason, J. O., et al. (1999). The transcription factor, Pax6, is required for cell proliferation and differentiation in the developing cerebral cortex. Cereb. Cortex 9, 627-635. doi: $10.1093 /$ cercor/9.6.627

Weber, K., Mock, U., Petrowitz, B., Bartsch, U., and Fehse, B. (2010). Lentiviral gene ontology (LeGO) vectors equipped with novel drug-selectable fluorescent proteins: new building blocks for cell marking and multi-gene analysis. Gene Ther. 17, 511-520. doi: 10.1038/gt.2009.149

$\mathrm{Xu}$, Q., Tam, M., and Anderson, S. A. (2008). Fate mapping Nkx2.1-lineage cells in the mouse telencephalon. J. Comp. Neurol. 506, 16-29. doi: 10.1002/cne.21529

Yu, K., McGlynn, S., and Matise, M. P. (2013). Floor plate-derived sonic hedgehog regulates glial and ependymal cell fates in the developing spinal cord. Development 140, 1594-1604. doi: 10.1242/dev.090845

Zhao, L., Zevallos, S. E., Rizzoti, K., Jeong, Y., Lovell-Badge, R., and Epstein, D. J. (2012). Disruption of SoxB1-dependent Sonic hedgehog expression in the hypothalamus causes septo-optic dysplasia. Dev. Cell 22, 585-596. doi: 10.1016/j.devcel.2011.12.023

Zhao, T., Szabo, N., Ma, J., Luo, L., Zhou, X., and Alvarez-Bolado, G. (2008). Genetic mapping of Foxb1-cell lineage shows migration from caudal diencephalon to telencephalon and lateral hypothalamus. Eur. J. Neurosci. 28, 1941-1955. doi: 10.1111/j.1460-9568.2008.06503.x

Zhao, T., Zhou, X., Szabo, N., Leitges, M., and Alvarez-Bolado, G. (2007). Foxb1driven Cre expression in somites and the neuroepithelium of diencephalon, brainstem, and spinal cord. Genesis 45, 781-787. doi: 10.1002/dvg.20356

Conflict of Interest Statement: The authors declare that the research was conducted in the absence of any commercial or financial relationships that could be construed as a potential conflict of interest.

Copyright (C) 2015 Haddad-Tóvolli, Paul, Zhang, Zhou, Theil, Puelles, Blaess and Alvarez-Bolado. This is an open-access article distributed under the terms of the Creative Commons Attribution License (CC BY). The use, distribution or reproduction in other forums is permitted, provided the original author(s) or licensor are credited and that the original publication in this journal is cited, in accordance with accepted academic practice. No use, distribution or reproduction is permitted which does not comply with these terms. 